\title{
Numerical computation for parallel plate thermoacoustic heat exchangers in standing wave oscillatory flow
}

\author{
A. Piccolo \\ Department of Civil Engineering, University of Messina \\ Contrada di Dio -98166 S. Agata (Messina), Italy
}

\begin{abstract}
A simplified computational method for studying the heat transfer characteristics of parallel plate thermoacoustic heat exchangers is presented. The model integrates the thermoacoustic equations of the standard linear theory into an energy balance-based numerical calculus scheme. Details of the time-averaged temperature and heat flux density distributions within a representative domain of the heat exchangers and adjoining stack are given. The effect of operation conditions and geometrical parameters on the heat exchanger performance is investigated and main conclusions relevant for HX design are drawn as far as fin length, fin spacing, blockage ratio, gas and secondary fluid-side heat transfer coefficients are concerned. Most relevant is that the fin length and spacing affect in conjunction the heat exchanger behaviour and have to be simultaneously optimized to minimize thermal losses localized at the HX-stack junctions. Model predictions fit experimental data found in literature within $36 \%$ and $49 \%$ respectively at moderate and high acoustic Reynolds numbers.
\end{abstract}

Key Words: Thermoacoustics, Heat exchanger, Heat, Sound, Finite differences Tel.: ++39 090 3977311, Fax: ++39 0903977480 email: apiccolo@unime.it 


\section{Nomenclature}

$a=$ speed of sound $\left(\mathrm{m} \mathrm{s}^{-1}\right)$

$A=$ surface area $\left(\mathrm{m}^{2}\right)$

$c_{P}=$ isobaric specific heat $\left(\mathrm{J} \mathrm{kg}^{-1} \mathrm{~K}^{-1}\right)$

$c_{F}=$ specific heat of the secondary fluid

$\left(\mathrm{Jkg}^{-1} \mathrm{~K}^{-1}\right)$

$D R=$ drive ratio $\left(=P_{A} / P_{0}\right)$

$\dot{\mathbf{e}}=\operatorname{energy}$ flux density $\left(\mathrm{Wm}^{-2}\right)$

$f=$ spatially averaged $h$ function

$h=$ thermoviscous function,

specific enthalpy $\left(\mathrm{J} \mathrm{m}^{-3}\right)$,

heat transfer coefficient $\left(\mathrm{Wm}^{2} \mathrm{~K}^{-1}\right)$

$j=$ imaginary unit

$\mathrm{j}_{\mathrm{C}}=$ Colburn-j factor

$k=$ wave number $\left(\mathrm{m}^{-1}\right)$

thermoviscous function (m)

$K=$ thermal conductivity $\left(\mathrm{Wm}^{-1} \mathrm{~K}^{-1}\right)$

$l=$ half plate/fin thickness $(\mathrm{m})$

$L=$ heat exchanger or stack length (m)

$\dot{m}=$ secondary fluid mass flux $\left(\mathrm{kg} \mathrm{s}^{-1}\right)$

Mol $=$ gas molecular weight $\left(\mathrm{kg} \mathrm{kmol}^{-1}\right)$

$\mathrm{Nu}=$ Nusselt number

$p=$ acoustic pressure $(\mathrm{Pa})$
$P=$ pressure $(\mathrm{Pa})$

$\operatorname{Pr}=$ Prandtl number

$P_{T}=$ internal perimeter of a tube section

(m)

$\dot{q}=$ time-averaged heat flux density

$\left(\mathrm{W} \mathrm{m}^{-2}\right)$

$\dot{Q}=$ heat load (W)

$\dot{Q}^{*}=$ heat flux at the fin surface (W)

$R=$ gas universal constant $\left(\mathrm{J} \mathrm{K}^{-1} \mathrm{kmol}^{-1}\right)$

$R^{\prime}=R \operatorname{Mol}^{-1}\left(\mathrm{~kJ} \mathrm{~K}^{-1} \mathrm{~kg}^{-1}\right)$

$t=$ time (s)

$T=$ temperature $(\mathrm{K})$

$u=$ specific internal energy $\left(\mathrm{J} \mathrm{m}^{-3}\right)$

$U=$ fin-to-reservoir unitary global

conductance $\left(\mathrm{Wm}^{-2} \mathrm{~K}^{-1}\right)$

$v=$ acoustic particle velocity $\left(\mathrm{m} \mathrm{s}^{-1}\right)$

$x=$ axial coordinate/direction $(\mathrm{m})$

$x_{s}=$ stack center coordinate $(\mathrm{m})$

$y=$ transverse coordinate/direction (m)

$y_{0}=$ half plate/fin spacing $(\mathrm{m})$

\section{Greek symbols}

$\alpha=$ fit constant of experimental values of $K_{S}, K_{C}$ and $K_{H}\left(\mathrm{~W} \mathrm{~m}^{-1} \mathrm{~K}^{-1}\right)$ 
$\beta=$ thermal expansion coefficient $\left(\mathrm{K}^{-1}\right)$

$\gamma=$ ratio of isobaric to isochoric specific

heat

$\delta=$ penetration depth, $\mathrm{m}$

$\eta=$ shear viscosity coefficient

$\left(\mathrm{N} \mathrm{m}^{-2} \mathrm{~s}^{-1}\right)$

$\theta=T_{F}-T_{0}(\mathrm{~K})$

$\Delta T_{H X S}=$ stack-HX $T$ difference $(\mathrm{K})$

$\Delta T_{H X g}=$ gas-HX $T$ difference $(\mathrm{K})$

$\kappa=$ gas thermal diffusivity $\left(\mathrm{m}^{2} \mathrm{~s}^{-1}\right)$

$\lambda=$ wavelength of the sound wave (m)

$\mu=h_{F} P_{T} / \dot{m} c_{F}$

$v=$ kinematic viscosity $\left(\mathrm{m}^{2} \mathrm{~s}^{-1}\right)$

$\xi=$ generic acoustic variable

$\Pi=$ thermoviscous function

$\rho=$ density of the gas $\left(\mathrm{kg} \mathrm{m}^{-3}\right)$

$\Phi=$ viscosity stress tensor $\left(\mathrm{N} \mathrm{m}^{-2} \mathrm{~s}^{-2}\right)$

$\chi=$ fit constant of experimental values of

$K_{S}, K_{C}$ and $K_{H}\left(\mathrm{~K}^{-1}\right)$

$\omega=\operatorname{angular}$ frequency $\left(\mathrm{rad} \mathrm{s}^{-1}\right)$

$\Omega=$ blockage ratio
$0=$ mean, time averaged

$1=$ first order acoustic variable

$A=$ acoustic amplitude at a pressure

antinode

$C=$ referred to the cold $\mathrm{HX}$

$F=$ secondary fluid

$H=$ referred to the hot $\mathrm{HX}$

in = evaluated at the inlet of the HX

$P=$ isobaric

res $=$ resonator

$r e f=$ reference value

$s=$ stack

solid $=$ solid

$x=$ longitudinal, $x$-component

$y=$ transversal, $y$-component

$z=$ along the $z$ direction

$\kappa=$ thermal

$v=$ viscous

\section{Superscripts}

in = evaluated at the inlet of the HX

$*=$ evaluated at the fin surface

\section{Subscripts}




\section{Introduction}

The enhanced use of renewable energies has nowadays become a major concern for the world energy policy to meet the target of sustainable development. Thermoacoustic (TA) technology can play a significant role in this context because of its remarkable advantages over conventional energetic technologies. TA engines are sound-heat energy conversion devices which operate with no moving components, use no-polluting working gases, can be powered by low-grade energetic inputs (waste heat, solar energy, etc.) and which join construction simplicity/reliability to low fabrication costs.

The physical key mechanism for these favourable technical features is the "thermoacoustic effect" where a sound wave naturally constrains fluid particles oscillating near solid surfaces to undergo properly phased pressure changes and heat transfers through a thermodynamic cycle $[1,2]$. The sound pressure-velocity phase angle (imposed by an acoustic network) distinguishes between travelling-wave and standing-wave engines. In the former the thermal interaction of the oscillating gas with the solid boundaries of a regenerator (an high heat capacity porous medium of small hydraulic radius) results in Stirling-type cycles with engine conversion efficiencies above $40 \%$ of the Carnot efficiency [3]. In the latter the fluid particles perform Brayton-type cycles in a stack (of larger pores) but "intrinsic" thermal irreversibilities limit the engine performances typically below $20 \%$ of Carnot's [3].

For the thermoacoustic effect to be fully exploitable in powerproduction/refrigeration applications the stack/regenerator must work in conjunction with a couple of heat exchangers (HXs) which transfer heat between its edges and external heat sources and sinks. These components have recently been the object of 
many research studies since a significant improvement of the overall engine's performance is expected to derive from their optimization. The finned-tube [4], and shell and tube [5] HX configurations are commonly used but reliable and univocal design criteria are still lacking. The main goal in designing efficient HXs is the achievement of high transfer rates in conjunction to low acoustic dissipation. Addressing this task for oscillatory fluid flows entails a series of new challenges compared to traditional compact HXs working in steady and unidirectional flows:

a) Longitudinal (along the $x$ direction parallel to the particle oscillation) and transverse (along the $y$ direction normal to the fin/plate surfaces) dimensions are bounded respectively by the particle displacement amplitude $x_{1}$ and by the thermal penetration depth $\delta_{\kappa}(=\sqrt{2 \kappa / \omega})$, the distance through which heat can diffuse in an acoustic cycle $(\kappa$ and $\omega$ being respectively the gas thermal diffusivity and the angular frequency of the sound wave). This imposes severe restrictions on the heat transfer area and on the flow resistance minimization.

b) The junctions between the stack/regenerator and the HXs are interested by complex temperature and flow patterns due to cross-sectional abrupt changes and entrance effects. $[6,7]$ These phenomena may strongly affect the actual heat transfer and power dissipation rates.

c) Unambiguous and reliable heat transfer correlations laws for the oscillatory flow regime in the sub-kHz domain are not yet available. Conventional steady flow correlations are commonly applied for the estimation of the heat transfer coefficients previous "ad-hoc" modifications $[8,9]$.

d) Large temperature differences are imposed by the hot and cold HXs to the ends of the stack/regenerator (typically long only several centimeters). These high temperature 
gradients immediately results in performance degradation (especially in TA refrigerators) due to the associated increased thermal irreversibilities. Temperature differences between the HXs and the stack/regenerator should be minimized preserving however an efficient inter-element heat exchange.

These issues cannot be faced by the standard linear theory [1,2] owing its 1D character and being based on the so called "mean field approximation" [10]. Thus 2D or 3D numerical and analytical models able to account for the transverse heat transfer phenomena taking place in the HXs are currently developed [11-15]. In this paper the results of a $2 \mathrm{D}$ numerical model for the study of the thermal performance of parallel plate thermoacoustic HXs coupled to a stack in a simplified configuration are presented. The model describes resonator acoustics through an idealized 1D sound wave while performs detailed $2 \mathrm{D}$ computations of the time-averaged temperature and energy flux distributions within a representative domain of the stack and HXs. These last are generated by an energy balance-based numerical calculus scheme which implements the energy transport variables of the classical linear thermoacoustic theory. The model constitutes an extended version of the one presented in $[8,16]$ (simulating a thermally isolated stack) by inclusion of hydrodynamic heat transport along the transverse $y$ direction, temperature dependent thermophysical gas/solid parameters, viscous dissipation and adjacent HXs interacting with "hot" and "cold" thermal reservoirs. The model is validated by comparing numerical predictions against experimental data found in literature. The effect of operating conditions and geometrical parameters on the heat transfer characteristics are investigated. Information on the optimal settings which maximize the HX performance is derived. 


\section{Formulation}

The thermoacoustic model system considered in this work is a stack of parallel plates, of length $L_{S}$, sandwiched between "hot" and "cold" parallel-fin HXs respectively of length $L_{H}$ and $L_{C}$. This assembly is located at a distance $x_{S}$ from the center of a halfwavelength $(\lambda / 2)$ gas filled resonator sustaining a standing acoustic wave as shown in Fig. 1. Stack plates and HX fins are modeled with the same spacing $\left(2 y_{0}\right)$ and thickness (2l) so the three structures result characterized by the same blockage ratio $\Omega=A_{s} / A_{\text {res }}=1 /\left(1+l / y_{0}\right)$, where $A_{\text {res }}$ is the cross sectional area of the resonator and $A_{s}$ is the cross sectional area of the stack/HXs open to gas flow. This choice, while allowing for a simple geometry, should lead to a reduction of the detriment effects discussed in section 1 at point $b$, being the flow and energy path almost not interrupted at the junctions. The implicit assumption is that an efficient HX is being modelled. The HX fins are set close to the ends of the stack plates but are not in touch with them in order to reduce adverse heat conduction leaks from hot to cold HX. To include in the calculation the effect of the heat transfer coefficient from the secondary fluid-side, the HXs are modeled to thermally interact with two thermal reservoirs, the "hot" reservoir at temperature $T_{H}$ and the "cold" reservoir at temperature $T_{C}$. Since we are considering a thermoacoustic device working in the refrigeration mode the former acts as a heat sink while the latter acts as a heat source.

In the rest of this section the basic energy balance equations on which the model relies upon are defined together with the expressions of the energy transport variables entering in them. Preliminarily, the main assumptions involved in the model are discussed. 


\subsection{Basic approximations and simplifying conditions}

The formulation relies on the following assumptions:

- The problem is two-dimensional;

- The acoustic approximation is valid (low Mach number regime) and any acoustic variable $\xi$ can expresses in complex notation by the conventional first-order expansion $\xi(x, y, t)=\xi_{0}(x, y)+\operatorname{Re}\left\{\xi_{1}(x, y) e^{j \omega t}\right\}$ where $t$ is the time, $j$ the imaginary unit, $\operatorname{Re}\{\}$ signifies the real part and where the mean value $\xi_{0}$ is real but the amplitude $\xi_{1}$ is complex to account for both magnitude and phase of the oscillation.

- The working fluid is a Newtonian gas with viscosity obeying the ideal gas state equation $P_{0} / \rho_{0}=R^{\prime} T_{0}$ where $P_{0}$ is the static pressure, $\rho_{0}$ the static density, $T_{0}$ the mean (time-averaged) temperature and $R^{\prime}=R / M o l$ ( $R$ being the universal constant of gases and $\mathrm{Mol}$ the molecular weight of the gas under study). Temperature dependence of the gas thermophysical properties $\beta$ (thermal expansion coefficient), $\eta$ (shear viscosity coefficient) and $K$ (thermal conductivity coefficient) are accounted for by the relations

$$
\beta=\frac{1}{T_{0}} \quad \eta=\eta_{r e f}\left(\frac{T_{0}}{T_{r e f}}\right)^{0.7} \quad K=K_{r e f}\left(\frac{T_{0}}{T_{r e f}}\right)^{0.7}
$$

$\eta_{\text {ref }}$ and $K_{\text {ref }}$ being the values of $\eta$ and $K$ at the reference temperature $T_{r e f}$.

- The stack is significantly shorter than the acoustic wavelength and is not intrusive. This allows the sound field to be retained as spatially uniform over the HXs/stack assembly and to be approximated in the neighbor (but also inside the stack as far as the 
pressure is concerned) by a $1 \mathrm{D}$ loseless standing wave:

$$
p_{1}=P_{A} \sin \left(k x_{s}\right)=p_{0} \quad v_{x 1}=j \frac{P_{A}}{\rho_{0} a} \cos \left(k x_{s}\right)=j v_{0}
$$

$p_{1}$ being the amplitude of the dynamic pressure, $P_{A}$ the amplitude of the dynamic pressure at a pressure antinode, $v_{x 1}$ the amplitude of the longitudinal particle velocity, $k$ the wave number $(k=2 \pi / \lambda)$ and $a$ the sound velocity.

- The standard thermoacoustic equations are considered for the amplitudes of the oscillating temperature $T_{1}$, longitudinal velocity $v_{x 1}$, transverse velocity gradient $\partial v_{x 1} / \partial y$ transverse velocity $v_{y 1}$ and transverse velocity gradient $\partial v_{y 1} / \partial y$ inside a gas pore. If the specific heats of the plate/fin materials are notably greater than the isobaric specific heat of the gas $c_{p}$ (negligible temperature oscillations in the solid), the first three Eqs. read as $[1,2]:$

$$
\begin{gathered}
T_{1}=\frac{1}{\rho_{0} c_{P}}\left(1-h_{\kappa}\right) p_{1}-\frac{1}{\rho_{0} \omega^{2}(1-\operatorname{Pr})} \frac{d p_{1}}{d x} \frac{\partial T_{0}}{\partial x}\left[\left(1-h_{\kappa}\right)-\operatorname{Pr}\left(1-h_{v}\right)\right] \\
v_{x 1}=\frac{j}{\omega \rho_{0}} \frac{d p_{1}}{d x}\left(1-h_{v}\right) \quad \frac{\partial v_{x 1}}{\partial y}=\frac{2}{\omega \rho_{0} \delta_{v}^{2}} \frac{d p_{1}}{d x} k_{v}
\end{gathered}
$$

where

$$
h_{\kappa}=\frac{\cosh \left[(1+j) y / \delta_{\kappa}\right]}{\cosh \left[(1+j) y_{0} / \delta_{\kappa}\right]} \quad h_{v}=\frac{\cosh \left[(1+j) y / \delta_{v}\right]}{\cosh \left[(1+j) y_{0} / \delta_{v}\right]}
$$

and

$$
k_{\kappa}=\frac{\delta_{\kappa}}{1+j} \frac{\sinh \left[(1+j) y / \delta_{\kappa}\right]}{\cosh \left[(1+j) y_{0} / \delta_{\kappa}\right]} \quad k_{v}=\frac{\delta_{v}}{1+j} \frac{\sinh \left[(1+j) y / \delta_{v}\right]}{\cosh \left[(1+j) y_{0} / \delta_{v}\right]}
$$


and where $\operatorname{Pr}$ is the Prandtl number, $\delta_{v}=\sqrt{2 v / \omega}$ the viscous penetration depth ( $v$ being the kinematic viscosity) and where $y=0$ is fixed at the center of the fluid gap.

The analytical expression of $v_{y 1}$ can be derived from the linearized equation of continuity

$$
j \omega \rho_{1}+\frac{\partial}{\partial x}\left(\rho_{0} v_{x 1}\right)+\frac{\partial}{\partial y}\left(\rho_{0} v_{y 1}\right)=0
$$

Neglecting the term $\left(\partial \rho_{0} / \partial y\right) v_{y 1}$ in comparison to the term $\left(\partial \rho_{0} / \partial x\right) v_{x 1}$, $\left(v_{y 1}\right.$ being expected to be of order $\delta_{v} / \lambda$ smaller than $v_{x 1}$ ) and taking into account the relations

$$
\frac{1}{\rho_{0}} \frac{\partial \rho_{0}}{\partial x}=-\frac{1}{T_{0}} \frac{\partial T_{0}}{\partial x} \quad \rho_{1}=-\rho_{0} \beta T_{1}+\frac{\gamma}{a^{2}} p
$$

( $\gamma$ being the ratio of isobaric to isochoric specific heat) equation (5) can be put into the form

$$
\frac{\partial v_{y 1}}{\partial y}=j \omega \beta T_{1}-j \frac{\gamma \omega}{\rho_{0} a^{2}} p_{1}-\frac{\partial v_{x 1}}{\partial x}+\beta \frac{\partial T_{0}}{\partial x} v_{x 1}
$$

Equations (3) and (4) can now be substituted into Eq. (6) for $T_{1}$ and $v_{x 1}$ while the axial velocity gradient can be deduced from the wave equation (Eq. A19 of ref.[1]). Making substitutions and rearranging (by also using the thermodynamic relation $c_{p}=\beta a^{2} /(\gamma-1)$ ) we obtain

$$
\frac{\partial v_{y 1}}{\partial y}=j \frac{\omega}{\rho_{0} a^{2}}\left\{\frac{\left[1+(\gamma-1) f_{\kappa}\right]\left(1-h_{v}\right)-\left[1+(\gamma-1) h_{\kappa}\right]\left(1-f_{v}\right)}{\left(1-f_{v}\right)}\right\} p_{1}
$$




$$
+j \frac{\beta}{\rho_{0} \omega(1-\operatorname{Pr})}\left[\frac{f_{v}\left(1-h_{\kappa}\right)-f_{\kappa}\left(1-h_{v}\right)+\left(h_{\kappa}-h_{v}\right)}{\left(1-f_{v}\right)}\right] \frac{\partial T_{0}}{\partial x} \frac{d p_{1}}{d x}
$$

whose integration between 0 e $y$ provides

$$
\begin{gathered}
v_{y 1}=j \frac{\omega}{\rho_{0} a^{2}}\left\{\frac{\left[1+(\gamma-1) f_{\kappa}\right]\left(y-k_{v}\right)-\left[y+(\gamma-1) k_{\kappa}\right]\left(1-f_{v}\right)}{\left(1-f_{v}\right)}\right\} p_{1}+ \\
j \frac{\beta}{\rho_{0} \omega(1-\operatorname{Pr})}\left\{\frac{f_{v}\left(y-k_{\kappa}\right)-f_{\kappa}\left(y-k_{v}\right)+\left(k_{\kappa}-k_{v}\right)}{\left(1-f_{v}\right)}\right\} \frac{\partial T_{0}}{\partial x} \frac{d p_{1}}{d x}
\end{gathered}
$$

where

$$
f_{\kappa}=\frac{\tanh \left[(1+j) y_{0} / \delta_{\kappa}\right]}{\left[(1+j) y_{0} / \delta_{\kappa}\right]} \quad f_{v}=\frac{\tanh \left[(1+j) y_{0} / \delta_{v}\right]}{\left[(1+j) y_{0} / \delta_{v}\right]}
$$

In Fig. 2 the profile of the real and imaginary parts of Eq. (8) together with its module is reported as a function of the transverse coordinate $y$ for parameters corresponding to run 1 of Table 1 . The transverse velocity vanishes both at mid channel $y=0$ (for symmetry) and at the wall surface $y=y_{0}$.

- The longitudinal pressure gradient $d p_{1} / d x$ along the stack/HXs assembly is calculated by imposing continuity of volume flow rate at the entrance of the HXs

$$
\frac{d p_{1}}{d x}=\frac{\rho_{0} \omega}{\left(1-f_{v}\right) \Omega} v_{0}
$$

We remark as the validity of the above derived equations could be invalidated at the pore ends of the stack or at the HX fins where the gas mean temperature is expected to 
become strongly $y$-dependent to allow net heat exchange with the solid surface. Equation (3), instead, was derived as solution of the heat transport equation involving a $y$-independent $T_{0}$. Analogously, invoking validity of Eqs. (4), (7) and (8) at the fin outer ends (facing the duct) corresponds to neglect any entrance effect which may be the source of minor losses. Results of the present study have thus to be appreciated as a function of these major limitations.

\subsection{Energy balance equation and energy fluxes in the gas}

Under the simplifying conditions outlined in the previous sub-section the gas flow dynamics remains completely described through Eqs. (2), (4), (7) (8) and (9). Meanwhile, the temperature field is governed by the general equation of energy conservation [17]

$$
\frac{\partial}{\partial t}\left(\frac{1}{2} \rho v^{2}+\rho u\right)=-\nabla \cdot \dot{\mathbf{e}}
$$

where $u$ is the specific internal energy and where $\dot{\mathbf{e}}$, the energy flux density, is defined as

$$
\dot{\mathbf{e}}=\rho \mathbf{v}\left(\frac{1}{2} v^{2}+h\right)-\mathbf{v} \cdot \mathbf{\Phi}-K \nabla T
$$

$h$ being the specific enthalpy and $\Phi$ the viscous stress tensor. Time averaging Eq. (10) over an acoustic cycle leads to

$$
\nabla \cdot \overline{\dot{\mathbf{e}}}=0
$$


where over-bar means time-averaged. Equation (12) is applied in this work to each computational cell of the simulation domain to impose local energy balance in the gas. To accomplish this, a finite difference technique is employed where the quantitative results of standard linear theory are considered for $x$ and $y$ the components of the timeaveraged energy flux density. These last are derived in the following subsections.

\subsubsection{Energy flux density along the $x$ direction}

The $x$ component of the time-averaged enthalpy flux density is [17]

$$
\overline{\dot{e}}_{x}=\frac{\omega}{2 \pi}\left(\int_{0}^{2 \pi / \omega} \rho v_{x}\left(\frac{1}{2} v^{2}+h\right) d t-\int_{0}^{2 \pi l \omega}\left(v_{x} \sigma_{x x}+v_{y} \tau_{x y}\right) d t-K \int_{0}^{2 \pi ! \omega} \frac{\partial T}{\partial x} d t\right)
$$

where the components of the viscous stress tensor are

$$
\begin{aligned}
& \sigma_{x x}=\frac{2}{3} \eta\left(2 \frac{\partial v_{x}}{\partial x}-\frac{\partial v_{y}}{\partial y}\right) \approx-\frac{2}{3} \eta \frac{\partial v_{y}}{\partial y} \\
& \tau_{x y}=\tau_{y x}=\eta\left(\frac{\partial v_{x}}{\partial y}+\frac{\partial v_{y}}{\partial x}\right) \approx \eta \frac{\partial v_{x}}{\partial y} \\
& \sigma_{y y}=\frac{2}{3} \eta\left(2 \frac{\partial v_{y}}{\partial y}-\frac{\partial v_{x}}{\partial x}\right) \approx \frac{4}{3} \eta \frac{\partial v_{y}}{\partial y}
\end{aligned}
$$

the $x$ velocity gradients having being neglected because of order $\delta_{v} / \lambda$ smaller than the $y$ velocity gradients. Although the second integral on the right hand side of Eq. (13) is 
generally neglected because including terms of second order in velocity, it is retained in this work because we are trying to identify the impact of loss mechanisms on the performance of the two HXs. Making use of the complex notation and retaining only terms up to second order Eq. (13) can be written as:

$$
\overline{\dot{e}}_{x}=\frac{1}{2} \rho_{0} c_{P} \operatorname{Re}\left\{T_{1} \widetilde{v}_{x 1}\right\}+\frac{1}{3} \eta \operatorname{Re}\left\{\frac{\partial v_{y 1}}{\partial y} \widetilde{v}_{x 1}\right\}-\frac{1}{2} \eta \operatorname{Re}\left\{\frac{\partial \widetilde{v}_{x 1}}{\partial y} v_{y 1}\right\}-K \frac{\partial T_{0}}{\partial x}
$$

where tilde indicates complex conjugation. Substituting now Eqs (2) and (9) into equations (3), (4) (7) and (8) and these last in Eq. (14), the following expression is found, at second order in the acoustic oscillation amplitude, for $\overline{\dot{e}}_{x}$ :

$$
\begin{aligned}
\dot{e}_{x}= & \frac{1}{2 \Omega} \operatorname{Im}\left\{\frac{\Pi_{1} \widetilde{\Pi}_{2}}{\left(1-\widetilde{f}_{v}\right)}\right\} P_{0} v_{0}-\frac{\rho_{0} c_{P}}{2 \omega \Omega^{2}(1-\operatorname{Pr})\left|1-f_{v}\right|^{2}} \frac{\partial T_{0}}{\partial x} \operatorname{Im}\left\{\Pi_{1} \widetilde{\Pi}_{2}\right\} v_{0}^{2}+ \\
& \frac{\eta \omega}{3 \Omega \rho_{0} a^{2}} \operatorname{Re}\left\{\frac{\widetilde{\Pi}_{2} \Pi_{5}}{\left(1-\widetilde{f}_{v}\right)}\right\} p_{0} v_{0}+\frac{\eta \beta}{3 \Omega^{2}(1-\operatorname{Pr})\left|1-f_{v}\right|^{2}} \frac{\partial T_{0}}{\partial x} \operatorname{Re}\left\{\widetilde{\Pi}_{2} \Pi_{6}\right\} v_{0}^{2}+ \\
& \frac{\eta \omega}{\rho_{0} \Omega a^{2} \delta_{v}^{2}} \operatorname{Im}\left\{\frac{\widetilde{k}_{v} \Pi_{3}}{\left(1-\widetilde{f}_{v}\right)}\right\} p_{0} v_{0}+\frac{\eta \beta}{\delta_{v}^{2} \Omega^{2}(1-\operatorname{Pr})\left|1-f_{v}\right|^{2}} \frac{\partial T_{0}}{\partial x} \operatorname{Im}\left\{\widetilde{k}_{v} \Pi_{4}\right\} v_{0}^{2}-K \frac{\partial T_{0}}{\partial x}
\end{aligned}
$$

where

$$
\begin{aligned}
& \Pi_{1}=\left(1-h_{\kappa}\right) \\
& \Pi_{2}=\left(1-h_{v}\right) \\
& \Pi_{3}=\frac{\left[1+(\gamma-1) f_{\kappa}\right]\left(y-k_{v}\right)-\left[y+(\gamma-1) k_{\kappa}\right]\left(1-f_{v}\right)}{\left(1-f_{v}\right)}
\end{aligned}
$$




$$
\begin{aligned}
& \Pi_{4}=\frac{f_{v}\left(y-k_{\kappa}\right)-f_{\kappa}\left(y-k_{v}\right)+\left(k_{\kappa}-k_{v}\right)}{\left(1-f_{v}\right)} \\
& \Pi_{5}=\frac{\left[1+(\gamma-1) f_{\kappa}\right]\left(1-h_{v}\right)-\left[1+(\gamma-1) h_{\kappa}\right]\left(1-f_{v}\right)}{\left(1-f_{v}\right)} \\
& \Pi_{6}=\frac{f_{v}\left(1-h_{\kappa}\right)-f_{\kappa}\left(1-h_{v}\right)+\left(h_{\kappa}-h_{v}\right)}{\left(1-f_{v}\right)}
\end{aligned}
$$

\subsubsection{Energy flux density along the y direction}

The time averaged hydrodynamic enthalpy flux along the transverse direction is

$$
\overline{\dot{e}}_{y}=\frac{\omega}{2 \pi}\left(\int_{0}^{2 \pi / \omega} \rho v_{y}\left(\frac{1}{2} v^{2}+h\right) d t-\int_{0}^{2 \pi t \omega}\left(v_{x} \tau_{x y}+v_{y} \sigma_{y y}\right) d t-K \int_{0}^{2 \pi t \omega} \frac{\partial T}{\partial y} d t\right)
$$

Proceeding analogously as done before for $\overline{\dot{e}}_{x}$ we obtain

$$
\overline{\dot{e}}_{y}=\frac{1}{2} \rho_{0} c_{P} \operatorname{Re}\left\{T_{1} \widetilde{v}_{y 1}\right\}-\frac{1}{2} \eta \operatorname{Re}\left\{\frac{\partial v_{x 1}}{\partial y} \widetilde{v}_{x 1}\right\}-\frac{2}{3} \eta \operatorname{Re}\left\{\frac{\partial v_{y 1}}{\partial y} \widetilde{v}_{y 1}\right\}-K \frac{\partial T_{0}}{\partial y}
$$

which, by substitution of the explicit expression of the acoustic variables, becomes

$$
\overline{\dot{e}}_{y}=\frac{\omega}{2 \rho_{0} a^{2}} \operatorname{Im}\left\{\Pi_{1} \widetilde{\Pi}_{3}\right\} p_{0}^{2}+\frac{\beta}{2 \Omega(1-\operatorname{Pr})} \operatorname{Im}\left\{\frac{\Pi_{1} \widetilde{\Pi}_{4}}{\left(1-\widetilde{f}_{v}\right)}\right\} \frac{\partial T_{0}}{\partial x} p_{0} v_{0}+
$$




$$
\begin{aligned}
& \frac{c_{p}}{2 \Omega(1-\operatorname{Pr}) a^{2}} \operatorname{Re}\left\{j \frac{\left(\Pi_{1}-\operatorname{Pr} \Pi_{2}\right) \widetilde{\Pi}_{3}}{\left(1-f_{v}\right)}\right\} \frac{\partial T_{0}}{\partial x} p_{0} v_{0}+ \\
& \frac{\beta \rho_{0} c_{p}}{2 \omega \Omega^{2}(1-\operatorname{Pr})^{2}\left|1-f_{v}\right|^{2}} \operatorname{Re}\left\{j\left(\Pi_{1}-\operatorname{Pr} \Pi_{2}\right) \widetilde{\Pi}_{4}\right\}\left(\frac{\partial T_{0}}{\partial x}\right)^{2} v_{0}^{2}- \\
& \frac{\eta}{\Omega^{2} \delta_{v}^{2}\left|1-f_{v}\right|^{2}} \operatorname{Im}\left\{k_{v} \widetilde{\Pi}_{2}\right\} v_{0}^{2}-\frac{2 \eta \omega^{2}}{3 \rho_{0}^{2} a^{4}} \operatorname{Re}\left\{\widetilde{\Pi}_{3} \Pi_{5}\right\} p_{0}^{2}- \\
& \frac{2 \eta \beta \omega}{3 \rho_{0} \Omega a^{2}(1-\operatorname{Pr})} \frac{\partial T_{0}}{\partial x} \operatorname{Re}\left\{\frac{\widetilde{\Pi}_{4} \Pi_{5}}{\left(1-\widetilde{f}_{v}\right)}+\frac{\widetilde{\Pi}_{3} \Pi_{6}}{\left(1-f_{v}\right)}\right\} p_{0} v_{0}- \\
& \frac{2 \eta \beta^{2}}{3 \Omega^{2}(1-\operatorname{Pr})^{2}\left|1-f_{v}\right|^{2}}\left(\frac{\partial T_{0}}{\partial x}\right)^{2} \operatorname{Re}\left\{\widetilde{\Pi}_{4} \Pi_{6}\right\} v_{0}^{2}-K \frac{\partial T_{0}}{\partial y}
\end{aligned}
$$

Note as in the expressions derived for $\overline{\dot{e}}_{x}$ and $\overline{\dot{e}}_{y}$ the dependence on the stack position inside the resonator is provided by $p_{0}$ and $v_{0}$. In the rest of this study these energy flux densities are identified to heat flux densities $\left(\overline{\dot{e}}_{x} \equiv \dot{q}_{x}, \overline{\dot{e}}_{y} \equiv \dot{q}_{y}\right)$ because in the short stack approximation the work flux contribution to the total energy flux is small [1] (the overbars having been neglected to simplify the notation).

\subsection{Energy balance equation and energy fluxes in the solid}

The energy equations in the gas are coupled to the ones in the solid through heat exchange at the solid surfaces. The analogue of equation (12) for the solid portions is

$$
\nabla \cdot \dot{\mathbf{q}}=0
$$


where

$$
\dot{q}_{x}=-K_{S} \frac{\partial T_{0}}{\partial x} \quad \dot{q}_{x}=-K_{H} \frac{\partial T_{0}}{\partial x} \quad \dot{q}_{x}=-K_{C} \frac{\partial T_{0}}{\partial x}
$$

and

$$
\dot{q}_{y}=-K_{S} \frac{\partial T_{0}}{\partial y} \quad \dot{q}_{y}=-K_{H} \frac{\partial T_{0}}{\partial y} \quad \dot{q}_{y}=-K_{C} \frac{\partial T_{0}}{\partial y}
$$

$K_{S}$ being is the thermal conductivity of the plate material and $K_{H}$ and $K_{C}$ the thermal conductivities respectively of the "hot" and "cold" HXs. The temperature dependence of these parameters is described by empirical laws of the form $K_{\text {solid }}=K_{\text {ref }}+\alpha \exp \left( \pm \chi T_{0}\right)$, with $K_{r e f}, \alpha$ and $\chi$ determined from the fit of experimental data found in literature.

\subsection{Energy balance in the heat exchangers}

The cooling power $\dot{Q}_{C}$ produced thermoacoustically in the stack is supposed to be extracted through the cold HX from a cold thermal reservoir at temperature $T_{C}$. Analogously, the sum of $\dot{Q}_{C}$ and of the heat produced by viscous dissipation over the surfaces of the stack/HXs assembly is rejected through the hot HX to a hot thermal reservoir at temperature $T_{H}$. The modeled HXs are of the flat tube type (as those considered in [18]) with secondary fluids flowing inside the tubes along the $z$ direction (see Figure 3). This configuration leads to a more simplified modeling compared to other geometries but introduces in the problem a $z$ dependence not included in our 2- $D$ $(x, y)$ treatment. To overcome this limitation we assume for the inner surface of the HXs 
tubing a constant temperature wall boundary condition (compatible with the high thermal conductivities of the HX material) but substitute for the local heat flux density normal to the inner tubing surface $\dot{q}_{y}(z)$ a proper mean value obtained by averaging $\dot{q}_{y}(z)$ over the length $L_{Z}$ of the tube. We start from the well-known expression of the temperature variation $T_{F}(z)$ of a fluid along a channel with constant wall temperature $T_{0}$

$$
\theta(z)=\theta_{i n} \exp (-\mu z)
$$

where $\theta(z)=T_{F}(z)-T_{0}, \theta_{i n}=T_{F}^{i n}-T_{0}$ and $\mu=h_{F} P_{T} / \dot{m} c_{F}$ ( $T_{F}^{\text {in }}$ being the inlet fluid temperature, $P_{T}$ the internal cross section perimeter of the tube, $h_{F}$ the convective coefficient fluidwall, $\dot{m}$ the mass flux and $c_{F}$ the fluid specific heat). The total heat flux exchanged by the secondary fluid is

$$
\dot{Q}=\int_{0}^{L_{z}} h_{F} P_{T} \theta(z) d z=\dot{m} c_{F}\left[1-\exp \left(-\mu L_{Z}\right)\right] \theta_{i n}
$$

So, the average heat flux density normal to the internal surface of the flat tube can be expressed in the form

$$
\dot{q}_{y}=\frac{\dot{Q}}{P_{T} L_{Z}}=U\left(T_{C}-T_{0}\right)
$$

where

$$
U=\frac{\dot{m} c_{F}}{P_{T} L_{Z}}\left[1-\exp \left(-\mu L_{Z}\right)\right]
$$

plays the role of a fin-to-reservoir unitary global conductance if $T_{\text {in }}$ is identified with $T_{C}$ 
or $T_{H}$ (in the hypothesis that the secondary fluid make perfect thermal contact with the thermal reservoirs). Equation (21) is used in this work to couple the heat flux thermoacoustically generated in the stack to the heat carried out by the secondary fluid.

\subsection{Numerical scheme}

The periodicity of the stack and HXs structures along $y$ allows calculations to be performed in a single channel of the stack/HXs assembly and in the couple of plates/fins enclosing it. The computational domain is further reduced by symmetry from half a gas duct to half a plate/fin as indicated in Fig. 4 by the light grey area together with the coordinate system used. The axis parallel to the plates is the $x$ axis; $x=0$ is chosen to be the beginning of the cold HX on the left. The $y$ axis is perpendicular to the stack-plates; $y=0$ is chosen to be the midpoint between the two adjacent plates or fins. The calculation of the steady-state two-dimensional time-averaged temperature distribution is performed using a finite difference methodology where temperature spatial gradients are discretized using first order nodal temperature differences. To this end, the computational domain is subdivided using a rectangular grid. In the $x$ direction the computation mesh size is typically $0.005 L_{s}$ while in the $y$ direction the computation mesh size is typically $0.02 y_{0}$. The following boundary conditions are imposed:

- Symmetry on nodal lines at $y=0$ and $y=y_{0}+l$

$$
\left(\frac{\partial T_{0}}{\partial y}\right)_{y=0}=\left(\frac{\partial T_{0}}{\partial y}\right)_{y=y_{0}+l}=0
$$


- continuity of temperature and transverse heat fluxes at the gas-solid interfaces $\left(y=y_{0}\right)$

$$
T_{0}\left(y=y_{0}\right)=\left.T_{0}\right|_{\text {solid }}\left(y=y_{0}\right) \quad K\left(\frac{\partial T_{0}}{\partial y}\right)_{y=y_{0}}=K_{\text {solid }}\left(\frac{\partial T_{0}}{\partial y}\right)_{y=y_{0}}
$$

where subscript "solid" refers in general both to the stack plate and the HX fins.

- continuity of transverse heat fluxes at the inner surface of the HX tubing:

$$
-K_{C}\left(\frac{\partial T_{0}}{\partial y}\right)_{\text {tube }}=U_{C}\left(T_{C}-T_{0}\right) \quad-K_{H}\left(\frac{\partial T_{0}}{\partial y}\right)_{\text {tube }}=U_{H}\left(T_{H}-T_{0}\right)
$$

- Vanishing heat flux at the solid HX fin terminations $\left(y_{0} \leq y \leq y_{0}+l\right)$ facing the duct

$$
\left(\frac{\partial T_{0}}{\partial x}\right)_{x=0}=\left(\frac{\partial T_{0}}{\partial x}\right)_{x=\left(L_{C}+L_{S}+L_{H}\right)}=0
$$

- Vanishing flux at the HX pore ends $\left(0 \leq y<y_{0}\right)$ facing the duct (gas oscillations outside the HXs are adiabatic and thus the thermoacoustic effect vanishes)

$$
\dot{q}_{x}(x=0)=\dot{q}_{x}\left(x=L_{C}+L_{S}+L_{H}\right)=0
$$

As for the separation gap between the HX fin and the stack edge, it is set equal to $\delta_{\kappa}$ and, as a further simplifying hypothesis, the slice of gas enclosed in it is assumed at rest. Substituting Eqs. (15) and (17) in Eq. (12) and Eqs. (19) and (20) in Eq. (18) to 
impose local energy balance in each cell of the computational grid, a system of quadratic algebraic equations in the unknown variable $T_{0}$ is generated. The system is solved by a code developed by the author in FORTRAN-90 language which executes the recursive Newton-Raphson method [19]. At each iteration the system of linear algebraic equations providing the temperature corrections for the next step is solved using a LU decomposition with partial pivoting and row interchanges matrix factorization routine. The latter is taken from the LAPACK library routines available online at [20], where details about accuracy, computation cost, etc. can be found. Once the time-averaged temperature distribution is known, it can be substituted in Eqs. (15) (17), (19) and (20) to determine the energy flux distributions along the $x$ and $y$ directions both in the gas and in the plate/fins. In addition, it can be used to calculate other variables of interest such as the energy dissipation, the entropy generation, etc.

Compared to other approaches currently used to simulate thermoacoustic devices the proposed model has the advantage of a reduced computational cost since it directly deals with time-averaged quantities and doesn't require integration of the conservation laws of mass, momentum and energy over the large number of acoustic cycles driving the system towards steady conditions. In section 3.5 the code is validated by comparing numerical predictions against experimental data found in literature.

\section{Results and discussion}

The performance of the coupled stack-HXs system is investigated at varying both operating conditions $\left(D R=P_{A} / P_{0}, x_{S}, U_{H}, U_{C}\right)$ and stack/fin geometry $\left(y_{0}, l, L_{H}, L_{C}\right)$. The 
parameters of different numerical simulations (runs) are listed in Table 1. In all tests helium at atmospheric pressure and at a mean temperature of $300 \mathrm{~K}$ is assumed as working fluid $\left(v=122 \times 10^{-6} \mathrm{~m}^{2} \mathrm{~s}^{-1}, K=0.152 \mathrm{Wm}^{-1} \mathrm{~K}^{-1}, a=1008 \mathrm{~ms}^{-1}, \mathrm{Pr}=0.68\right.$ at $T_{\text {ref }}=300$ $\mathrm{K})$. It is considered enclosed in a half-wavelength resonator (acoustic wavelength $\lambda=5.04 \mathrm{~m}$ ) having a resonance frequency of $200 \mathrm{~Hz}$; The stack is $0.07 \mathrm{~m}$ long and is constituted by stainless steel $\left(K_{S}=14.9 \mathrm{Wm}^{-1} \mathrm{~K}^{-1}\right.$ at $\left.T_{\text {ref }}=300 \mathrm{~K}\right)$ while the HXs material is copper $\left(K_{C}=K_{H}=401 \mathrm{Wm}^{-1} \mathrm{~K}^{-1}\right.$ at $\left.T_{r e f}=300 \mathrm{~K}\right)$. In all cases the temperature of the cold and hot reservoirs are fixed respectively to $T_{C}=297 \mathrm{~K}$ and $T_{H}=300 \mathrm{~K}$. The dynamic Reynolds number $\left(v_{x 1} \delta_{v} / v\right)$ and Mach number $\left(P_{A} / \rho_{0} a^{2}\right)$ resulted always smaller respectively than 500 and 0.1 , the values over which turbulence an non-linear behaviour are expected to affect fluid dynamics $[1,21]$.

The cooling power, $\dot{Q}_{C}$, is calculated by integrating $\dot{q}_{y}$ over the inner tubing surface of the cold HX

$$
\dot{Q}_{C}=\int_{0}^{L_{C}} U_{C}\left[T_{C}-T_{0}(x)\right] d x
$$

with $L_{Z}$ arbitrarily set to $1 \mathrm{~m}$. This quantity differs from the heat flux entering/leaving the outer fin surface

$$
\dot{Q}_{C}^{*}=\int_{0}^{L_{C}} K\left(\frac{\partial T_{0}}{\partial y}\right)_{y_{0}} d x
$$

by the heat flux transferred through the vertical fin surface facing the stack edge. Analogous quantities are calculated at the hot HX fin. At the operating conditions 
considered in this work $\dot{Q}_{C}$ resulted smaller than $\dot{Q}_{C}^{*}$ typically by $3 \%$, evidencing as the stack performance is degraded by the heat conduction losses at the stack-HX junctions.

\subsection{Effect of temperature difference}

Figure 5 illustrates the longitudinal temperature profile evaluated in correspondence of the centreline $\left(y=y_{0}+l\right)$ of the plate and of both fins for a drive ratio $D R\left(=P_{A} / P_{0}\right)=4.93$ $\%$ (run 2). The temperature distribution is linear in the stack (except at the stack edges) becoming flat in the fins, consistently with their high thermal conductivity (near isothermal fins). Temperature discontinuities $\Delta T_{H X S}$ are observed between the fins and the stack edges (with the stack temperature higher than the cold fin temperature) in accordance with experiments [22]. At low $U$ values $(U<100) \Delta T_{H X S}$ increases at increasing drive ratios as shown in the insert of the same figure (run 3). This behaviour can be interpreted observing that in the present model the HXs are thermally interacting with hot and cold reservoirs. So, depending on the magnitude of the fin-to-reservoir overall heat transfer coefficient $U$, the cold fin must cool down below $T_{C}$ and the hot fin must heat up above $T_{H}$ until the heat flux extracted from the cold reservoir and the one delivered to the hot reservoir balance the heat flux thermoacoustically pumped along the stack. At low $U$ values this causes an increase of the mean longitudinal temperature gradient across the HXs-stack assembly and thus of the temperature discontinuities observed at the HXs-stack junctions. The same figure shows as the effect becomes less marked at increasing $U$ values (run 4) till to invert its trend $\left(\Delta T_{H X S}\right.$ decreases with $D R$ ) for $U>100$ (run 5). In this latter case, in fact, the temperatures of the hot and cold HXs 
are "anchored" respectively to $T_{H}$ and $T_{C}$ and the enhanced hydrodynamic enthalpy flux activated by increasing $D R$ are compatible with lower axial temperature gradients. This is clearly shown in Fig. 6 where the cooling load results to be a decreasing (linear) function of the ratio between the temperature of the cold $\mathrm{HX}$ and the temperature of the hot $\mathrm{HX}$ (runs 6-9). Figure 5 also reveals that at high $U$ values $\Delta T_{H X S}$ vanishes in correspondence of a given drive ratio and then becomes negative (stack temperature lower than fin temperature). This circumstance, also experimentally evidenced by Mozurkewich [9], could allow for minimization of the detriment heat leakage phenomenon from hot to cold HX discussed in the previous sub-section. The $U$ magnitude, therefore, directly affects the temperature difference across the stack and this in turn influences the axial hydrodynamic enthalpy flux, i.e. ultimately, the cooling load. High $U$ values are desirable to improve the performance of the device, as evidenced in Fig. 7 where the growth of the cooling load with $U$ at selected $D R$ is observable (runs $7,9,10,11$ ).

The existence of inter-element heat transfer under zero temperature difference is not surprising because the driving temperature difference for heat extraction/immission at the HXs is the one between the HXs and the adjacent gas. High values of this transverse temperature gradient should be avoided, however, because they could enhance the thermal irreversibility associated to the heat transfer process and, in addition, could determine a steep axial temperature gradient in the gas which, as argued before, reduces the useful cooling load. In Fig. 8 the mean gas-fin temperature difference $\Delta T_{H X g}=<T_{H X C}>-<T_{g C}>$ at the cold $\mathrm{HX}$ is plotted as a function of $D R$ at selected $y_{0}$ values (runs 12, 13). Quantities $\left\langle T_{H X C}\right\rangle$ and $\left\langle T_{g C}\right\rangle$ represent the spatially averaged temperatures respectively of the cold HX (evaluated at the gas-solid interface $y=y_{0}$ ) and 
of the gas (evaluated at the centerline of the gas channel $y=0$ ):

$$
<T_{H X C}>=\frac{1}{L_{C}} \int_{0}^{L_{C}} T_{0}\left(x, y_{0}\right) d x \quad<T_{g C}>=\frac{1}{L_{C}} \int_{0}^{L_{C}} T_{0}(x, 0) d x
$$

The plot clearly shows the existence of two regimes: one at low $D R$ where $\Delta T_{H X g}$ exhibits a squared dependence on $D R\left(\Delta T_{H X g} \propto D R^{2}\right)$ and one at high $D R$ where $\Delta T_{H X g}$ depends linearly on $D R\left(\Delta T_{H X g} \propto D R\right)$. This result seems to conflict with the statements of Swift [23] which predicts rather a linear behaviour at low $D R$ and a squared-one at high $D R$. The disagreement can be explained analysing the Swift's predictive formula based of the "thermal boundary-layer" theory [23]:

$$
\Delta T_{H X g}=\frac{\dot{Q}_{C} \delta_{\kappa}}{K A}
$$

$A\left(=L_{C} L_{Z}\right)$ being the heat transfer surface area. At low $D R$ (for $\left.2 x_{1}<<L_{C}\right)$ it is expected $\dot{Q}_{C} \propto D R^{2}$ so if $2 x_{1}(\propto D R)$ is substituted for $L_{C}$ in $A$ (as done in [23]) the linear behavior is found while if $A$ is set always constant to $L_{C} L_{Z}$ (as done in the present work) the quadratic behavior is obtained. At high $D R$ (for $2 x_{1}>>L_{C}$ ) if the functional relationship $\dot{Q}_{C} \propto D R^{2}$ is retained (as done in [23]) and $A$ is set equal to $L_{C} L_{Z}$ the quadratic behavior is found but if the dependence of $\dot{Q}_{C}$ on $D R$ is not quadratic a deviation from the attended behaviour should be expected. This is indeed the case of the present study where simulations predict in the high drive ratio regime (when $2 x_{1}>>L_{C}$ ) a roughly 
linear dependence of $\dot{Q}_{C}$ on $D R$ (see next sub-section).

An additional important information that can be drawn from Fig. 8 is that the choice $y_{0} / \delta_{\kappa}=1.5$ in conjunction with $U=2000 \mathrm{Wm}^{-2} \mathrm{~K}^{-1}$ entails lower transverse temperature gradients and higher cooling loads (results not shown) compared to the other. So for a given HX length, the benefit of reduced thermal irreversibilities could be met in conjunction with enhanced cooling loads for suitable HX configurations.

\subsection{Effect of drive ratio}

To investigate the effect of drive ratio on the cooling load $\dot{Q}_{C}$ numerical simulations are performed at fixed fin lengths $L_{C}$ and $L_{H}$. Results are shown in Fig. 9 where the mean heat loads per unit fin area $\dot{q}_{C}$ and $\dot{q}_{H}$ (and their difference in the insert) are plotted as a function of $D R$ (run 15). The simulations reveal that at low drive ratios $\left(2 x_{1}<<L_{C}, L_{H}\right)$ both $\dot{q}_{C}$ and $\dot{q}_{H}$ increase proportionally to $D R^{2}$ as stated by the linear theory. At high drive ratios $\left(2 x_{1}>>L_{C}, L_{H}\right)$, however, the behavior of the heat loads differentiates: while $\dot{q}_{H}$ continues to grow faster than linearly (even if with a reduced strength), $\dot{q}_{C}$ exhibits a roughly linear dependence on $D R$. As already pointed out by Swift [23], a candidate cause for the appearance of the linear behavior could be the inefficiency of the HXs in transferring the full thermal load at high pressure amplitudes. This should happen when the peak to peak particle displacement amplitude exceeds the length of the heat exchangers. Although to the knowledge of the author there is no clear experimental evidence for this transition effect in literature, some analogue findings are reported in 
the numerical studies of Worlikar and Knio [12] and of Marx and Blanc-Bennon [24]. The last authors, in particular, observed the same transition in behavior in the acoustically stimulated hydrodynamic energy flux flowing within the gas pores of an isolated stack (without heat exchangers). It is interesting to note as the effect (attributed by the authors to thermal wave anharmonicity) appeared at the drive ratio for which the peak-to-peak particle displacement amplitude reached half the plate length. This condition could be retained as equivalent to the analogue put forward here for the HXs when considering that the plate edges of an isolated stack act as real HX fins and that at the operation point of the transition the stack may be considered as constituted by two HX fins (each of length $L_{S} / 2$ ) set in close contiguity.

As reported above, the analogous approaching of a linear behavior is not as evident in $\dot{q}_{H}$ as in $\dot{q}_{C}$. This fact could be explained observing that the hot HX have to sustain, in addition to the heat flux thermoacoustically pumped within the stack, the heat generated by viscous dissipation over all the solid surfaces of the channel (both stack and HXs). This contribute, which accounts for the larger heat transfer area of the hot HX compared to the cold one, has a well-known quadratic dependence on $D R$ and should correspond in our simplified model to the difference $\dot{q}_{H}-\dot{q}_{C}$. This last is plotted in the insert of Fig. 9 where the quadratic dependence on $D R$ is clearly observable.

\subsection{Effect of HX length and fin interspacing}

The HX length along the axial direction is a critical parameter in HX design because it determines the available heat transfer area and also accounts for the rate of viscous 
dissipation. To study the effect of the cold HX length $L_{C}$ on the cooling load $\dot{Q}_{C}$, simulations are performed at fixed stack position and blockage ratio and at varying $D R$, $y_{0}, L_{C}$ and $L_{H}$ values. A typical result is shown in Fig. 10 where $\dot{Q}_{C}$ is plotted as a function of $L_{C}$ at selected $D R$ values (run 15-17). Simulations reveal that $\dot{Q}_{C}$ increases quickly in the range $0<L_{C}<x_{1}$ becoming almost constant for $L_{C}>x_{1}$. At the considered operating conditions and for $L_{C} / 2 x_{1} \approx 1$ the cooling load amounts at about $93 \%$ of the maximum cooling load evaluated by calculating the total longitudinal enthalpy flux at the midpoint of the stack (a choice justified by the fact that the stack plate is longer than the particle displacement for all the $D R$ values considered). As pointed out by Swift [1], this should be the optimal condition for efficient inter-element heat transfer. This conclusion is based on the argument that a particle can transport heat over a distance not exceeding $2 x_{1}$ and that the total amount of particles able to participate to the interelement heat transfer corresponds to the gas portion comprised within a distance $2 x_{1}$ from the stack edge. We remarkably note as for all the considered drive ratios halving the plate length starting from the above invoked optimal value $\left(2 x_{1} \rightarrow x_{1}\right)$ produces a reduction of $\dot{Q}_{C}$ of less than $1 \%$ (from about 93 to $92 \%$ ). This result indicates, as already highlighted by Hofler [25], that the fin length could be chosen substantially shorter than the peak-to-peak particle displacement amplitude without compromising the HX performance and with great benefits in terms of viscous losses reduction. An experimental evidence for this finding can be found in [23] where it was recognized that doubling the length of the cold HX of a TA engine did not enhanced performance, even when the displacement amplitude much exceeded the length of the single HX.

The dependence of the cooling load $\dot{Q}_{C}$ on the plate/fin spacing $2 y_{0}$ at selected $L_{C}$ 
values is shown in Fig. 11 (runs 18-23). At a fixed $y_{0}$, the curve magnitude grows at increasing the fin length but the growth rate reduces with $L_{C}$ until the curves are being overlapping each other for high enough $L_{C}$ values. This trend is consistent with the results shown in Fig. 10 and should correspond to the flat portion of the curves attained for very long fins $\left(L_{C}>>x_{1}\right)$. Most important is that all curves peak at a well-defined value of the fin interspacing. The peak location results to be a slightly increasing (linear) function of the fin length, as evidenced in Fig. 11 by the straight line passing through the locus of maxima. This should correspond to a reduction of the effective length available for heat transfer (i.e. of the HX length) for short plate spacing $\left(y_{0} / \delta_{\kappa}<1\right)$. The optimum fin/plate spacing ranges between $2 y_{0}=2.98 \delta_{\kappa}\left(\right.$ for $\left.L_{C} / 2 x_{1}=0.079\right)$ and $2 y_{0}=3.34 \delta_{\kappa}$ (for $L_{C} / 2 x_{1}=1.9$ ) for $\Omega=0.76$. These results agrees with the general design procedure of selecting the plate spacing of the stack of thermoacoustic refrigerators in the interval $2 \delta_{\kappa} \leq 2 y_{0} \leq 4 \delta_{\kappa}[26]$.

The difference between the cooling load and the total axial enthalpy flux evaluated at the midpoint of the stack (maximum cooling load) reveals that the HX performance is affected by some kind of thermal losses. To get insight into this issue the transverse component of the heat flux density at the gas-solid interface $\left(y=y_{0}\right)$ is plotted in Fig. 12 as a function of the axial coordinate (runs 24,25$)$. In both the upper $\left(L_{C} / 2 x_{1}=0.53\right)$ and the lower $\left(L_{C} / 2 x_{1}=2.25\right)$ curve illustrated the heat flux exhibits a sharp peak near the fin ends facing the duct (outer ends). These are therefore the main areas where a net heat exchange between the fluid and the solid takes place The lower plot evidences as the heat flux density inverts its sign near the fin ends facing the stack (inner ends) of both cold and hot HX giving rise to a reverse flux which decreases the net heat transfer between the gas and the HXs, thus worsening the HX performance. Smaller transverse 
heat fluxes, qualitatively similar to those observed in thermoacoustic couples [27], are also found near the plate ends but they quickly vanish in the middle plate regions. The extent of the reverse heat fluxes, which are qualitatively similar to those found by Besnoin and Knio $[13,14]$, reduces with $D R$ till to vanish at high drive ratios when $L_{C} / 2 x_{1}<<1$, as evidenced by the upper curve of Fig. 12. An analogous trend is observed if $D R$ is held fixed and $L_{C}$ is progressively reduced. The reverse flux effect or, in general, the poor efficiency of the fin areas facing the stack (inner ends) in transferring heat could account for the previously discussed high performance of HXs whose length is considerably smaller than $2 x_{1}$. Reducing the length of the HX, in fact, results in decreasing the fin areas of poor efficiency (inner ends) so, even if the available heat transfer area becomes smaller, the heat transferred per unit area is increased. This is clearly observable in Fig 13 . where the mean heat flux per unit surface area $\left(\dot{Q}_{C}^{*} / L_{C}\right)$ exchanged by the cold fin with the gas is plotted as a function of the fin length at selected drive ratios (run 15-17). The area over which the HX is affected by the reverse flux also varies with the fin/plate spacing as illustrated in Fig. 14 (runs 18, 19, 21) where the longitudinal profile of the transverse heat flux density at the gas-solid interface $\left(y=y_{0}\right)$ of the cold HX fin is plotted at selected $y_{0}$ values. The comparative analysis of this figure with Fig. 11 informs that the points where $\dot{Q}_{C}$ peaks are almost coincident with the ones for which the reverse flux area results minimized. The lower plot of Fig. 12 reveals however that even when the reverse flux is eliminated, minor transverse heat fluxes localized at the stack edges persist.

The thermal losses above discussed affecting the HX-stack junction seems therefore to constitute a permanent feature of the inter-element heat transfer process and cannot be totally avoided. The results of the present study informs however that their strength 
can be minimized by suitable choices of the fin length and spacing which thus have to be simultaneously optimized.

\subsection{Effect of blockage ratio}

The analysis performed in the last section indicates that the optimum value of plate/fin spacing for which the cooling load peaks is around $3 \delta_{\kappa}$. This result was derived considering a constant blockage ratio, that is, in each run the plate thickness was "adjusted" to give rise for the selected $y_{0}$ to the constant value $\Omega=0.76$. The analysis, therefore, didn't take into account the effect of varying plate/fin spacing on particle axial velocity and effective cross sectional area available for heat transport. In order to estimate the effect of blocked ratio on the total cooling load simulations are performed at varying $y_{0}$ for a fixed value of the plate thickness $l$ (roughly equal to the thermal penetration depth in the fin material: $0.0005 \mathrm{~m}$ ). Also, the total number of channels comprised in a cross section of the stack are calculated assuming that this last fill in all the resonator cross-section, taken for simplicity quadratic in shape and of unitary surface area. The total cooling load sustained by the stack is then obtained by

multiplying $2 \dot{Q}_{C}$ for the resulting number of channels. Results are shown in Fig. 15 where the total cooling load is reported as a function of $y_{0}$ (and $\Omega$ ) at selected $L_{\mathrm{C}}$ values (run 26-28). All curves result much more peaked compared to the ones reported in Fig. 11 and the peak location (also in this case slightly dependent on $L_{C}$ ) falls at lower $y_{0}$ values, i.e., at a fin/plate interspacing roughly equal to $2 \delta_{\kappa}$ (to which the optimal value $\Omega \approx 0.59$ corresponds). The different behaviour can be interpreted observing that the 
lower the plate interspacing the higher the number of channels of the stack. This compensates for the decreasing of the heat flux pumped in a single channel which is observed in Fig. 11 to take place for $y_{0}<1.5 \delta_{\kappa}$. Simulations reveal that a change in plate thickness modifies the peak height and associated optimum blockage ratio but not the peak location. For example, doubling the plate thickness (for $L_{C} / 2 x_{1}=1$ ) reduces the peak height of about $9 \%$ and the optimal $\Omega$ by about $29 \%$ leaving the peak location unchanged (results not shown). Based on the results of this section the optimal plate/fin interspacing in stack-based thermoacoustic device should fall in the narrower range $2 \delta_{\kappa} \leq 2 y_{0} \leq 3 \delta_{\kappa}$.

\subsection{Derivation of heat transfer correlations}

In this section simulations are performed with the intent to develop heat transfer correlations which could be applied for the estimation of the gas-side heat transfer coefficient $h$ at the HX walls. At the same time, the comparison of the theoretical predictions against experimental data found in literature is considered as model validation.

Due to the existence of reverse heat flux localized at the inner edges of the HX fins the area-averaged $h$ values over the cold HX are computed as indicated in [13] by the formula

$$
h=\frac{<\dot{q}_{y}>}{<T_{H X C}>-<T_{g C}>}
$$

where 


$$
<\dot{q}_{y}>=\frac{1}{L_{C}} \int_{0}^{L_{C}} \dot{q}_{y}\left(x, y_{0}\right) d x
$$

Analogous formulas have been applied to the hot HX. The $h$ data thus obtained are converted to non-dimensional Nusselt numbers based on the hydraulic diameter of the pore $D_{h}\left(=4 y_{0}\right)$ and then to Colburn- $j$ factors

$$
\mathrm{Nu}_{D}=\frac{h D_{h}}{K} \quad \mathrm{j}_{C, D}=\frac{\mathrm{Nu}_{D}}{\operatorname{Re}_{1, D} \operatorname{Pr}^{1 / 3}}
$$

$\operatorname{Re}_{1, D}\left[=v_{x 1}(0) D_{h} / v\right]$ being the acoustic Reynolds number defined in terms of $D_{h}$ and of the amplitude of the acoustic velocity evaluated at gas centreline. The results of about 40 simulations (runs 29-37) carried out at varying $D R, L_{C}, L_{H}, y_{0}$, and $x_{S}$ values are illustrated in Fig. 16 where the resulting Colburn-j factors are plotted as a function of $\operatorname{Re}_{1, D}$. In the same graph the experimental measurements of Nsofor et al. [28], Peak et al. [29], Mozurkewich [9] and Brewster et al. [22] are reported. All HXs involved in these works are of the finned-tube type (except the one in [9] consisting of a copper screen soldered to tubes referred to by the author as "configuration C") so the measured $h$ values therein reported are considered suitable to make comparison with the predictions of the proposed model. Preliminary, in order to make the different set of data consistent, the experimental Nusselt and Reynolds numbers have been re-scaled by the same length parameter $\left(4 y_{0}\right)$. In addition, the rms velocity amplitudes entering in the Reynolds numbers reported in [5] have been "corrected" by the factor $\sqrt{2}$ and by the blockage ratio $\Omega$. 
The mean deviation of the predicted $\mathrm{j}_{C}$ values from the experimental ones amounts to $49 \%$ for the data of Peak et al., 36\% for the data o Brewster et al., $36 \%$ for the data of Mozurkewich and 56\% for the data of Nsofor et al. Deviations in the moderate and high Reynolds number regime $\left(\operatorname{Re}_{1}>500\right)$ are expected because of the increased weight of entrance/exit effects at the resonator-HX cross section interface. These effects are responsible of complex non-linear temperature and flow patterns (turbulent and vorticity flows [6].) which highly affect heat transfer rates causing, among other things, minor losses and heat leakage to the duct. The high deviation found at low $\operatorname{Re}_{1}$ numbers $\left(\operatorname{Re}_{1}<500\right)$ between simulations and experimental results of Nsofor et al. is quite surprising because the linear theory, on which the present model relies upon, is generally observed to match experiments quite well at low velocity amplitudes. In the evaluation of these results it has to be pointed out however that, although proper scaling procedures have been applied, the operating and geometrical conditions specified in the simulations are different from those of the selected experiments. It's remarkable to note, anyway, that al low $\operatorname{Re}_{1}$ values $\left(\operatorname{Re}_{1}<200\right)$ the simulation points match exactly the correlation curve derived by Peak et al. from the fit of their data. On the same curve, on the other hand, the data of Mozurkewich tend to collapse at decreasing $\operatorname{Re}_{1}$.

\section{Conclusions}

The performance of parallel-fin thermoacoustic HXs coupled to a stack in a simplified configuration is investigated through a 2D numerical model based on the classical linear thermoacoustic theory. Details of the transverse heat transfer at the HXs are highlighted 
and optimization information for their design are derived. The following conclusion can be drawn:

1. The magnitude of the heat transfer coefficient from the secondary fluid-side $(U)$ should be fully taken into account because it affects cooling load through its influence on the temperature distribution across the stack. High $U$ values are desirable to improve the performance of the device.

2. Fin length along the axial direction of particle oscillation can be chosen considerably lower than the peak-to-peak displacement amplitude without compromising the HX performance and with great benefits in terms of viscous losses reduction.

3. Thermal losses (reverse heat fluxes) localized at the stack-HX junctions degrade the HXs performance reducing the useful cooling load. These effects could account for about $7 \%$ of the deviations found between predictions of the linear theory and experimental measurements. Suitable choices of the fin spacing and fin length can contribute to minimize this detriment effects enhancing the effectiveness of the HXs.

4. Optimum fin/plate interspacing should fall in the range $2 \delta_{\kappa} \leq 2 y_{0} \leq 4 \delta_{\kappa}$. Selection of the best value is case dependent and should also take into account the simultaneous effect of blockage ratio, fin length and fin thickness.

5. Heat transfer coefficients from the gas-side can be predicted with a confidence of $36 \%$ and $49 \%$ respectively at moderate and high acoustic Reynolds numbers. The high deviation found at low $\mathrm{Re}_{1}$ with available experimental data requires further investigation.

The simplified computational method proposed in this work suffers by several limitations. In its current form the model is restricted to the acoustic and short stack approximations. Furthermore, the HXs are modeled as coupled to the stack in a 
simplified configuration while their arrangement in real TA devices can be more complex. The heat transport equations implemented in the code relies on the classical form of the function $T_{1}$ whose accuracy is questionable near the pore terminations. The model does not take into account for entrance and exit effects which may become relevant near sudden changes of cross section like those existing at the ends of the HXs. Further analysis is needed, particularly of the fluid and energy flows near the HX pore ends, for overcoming these shortcomings.

\section{Acknowledgments}

This research is financially supported by the EC under the project framed within the SEVENTH FRAMEWORK PROGRAMME “THermoacoustic Technology for Energy Applications" (Grant Agreement no.: 226415, Thematic Priority: FP7-ENERGY-2008FET, Acronym: THATEA).

The author also gratefully acknowledges the allocation of computer time from the "Centro di Calcolo Elettronico Attilio Villari" of the University of Messina. 


\section{References}

[1] G.W. Swift, Thermoacoustic engines, Journal of the Acoustical Society of America 84 (1988) 1145-1180.

[2] G.W. Swift, Thermoacoustics: A unifying perspective for some engines and refrigerators, Acoustical Society of America, 2002.

[3] S. Backhaus, G.W. Swift, A thermoacoustic Stirling heat engine: detailed study, Journal of the Acoustical Society of America 107 (2000) 3148-3166.

[4] K. Tang, R. Bao, G.B. Chen, Y. Qiu, L. Shou, Z.J. Huong, T. Jin, Thermoacoustically driven pulse tube cooler below 60 K, Cryogenics 47 (9-10) (2007) $526-529$.

[5] M.E.H. Tijani, S. Spoelstra, Study of coaxial thermoacoustic Stirling cooler. Cryogenics, 48 (2008) 77-82.

[6] L. Shi, Z.B. Yu, A.J. Jaworski, Vortex shedding flow patterns and their transitions in oscillatory flows past parallel-plate thermoacoustic stacks. Experimental Thermal and Fluid Science 34 (7) (2010) 954-965.

[7] A. Berson, M. Michard, P. Blanc-Benon, Measurement of acoustic velocity in the stack of a thermoacoustic refrigerator using particle image velocimetry, Heat and Mass Transfer 44 (8) (2008) 1015-1023.

[8] A. Piccolo, G. Pistone. Estimation of heat transfer coefficients in oscillating flows: the thermoacoustic case, International Journal of Heat and Mass Transfer 49 (9-10) 2006, 1631-1642.

[9] G. Mozurkewich, Heat transfer from transverse tubes adjacent to a thermoacoustic stack, Journal of the Acoustical Society of America 110 (2) (2001) 841-847. 
[10] V. Gusev, P. Lotton, H. Bailliet, S. Job, M. Bruneau, Relaxation-time approximation for analytical evaluation of temperature field in thermoacoustic stack. Journal of Sound and Vibration, 235 (5) (2000) 711-726.

[11] G. Mozurkewich, A model for transverse heat transfer in themoacoustics, Journal of the Acoustical Society of America 103 (1998) 3318-3326.

[12] A.S. Worlikar, O.M. Knio, Numerical study of oscillatory flow and heat transfer in a loaded thermoacoustic stack, Numerical Heat Transfer Part A 35 (1999) 49-65.

[13] E. Besnoin, O.M Knio, Numerical study of thermoacoustic heat exchangers in the thin plate limit, Numerical Heat Transfer A 40 (2001) 445-471.

[14] E. Besnoin, O.M Knio, Numerical study of thermoacoustic heat exchangers. Acta Acustica United with Acustica 90 (2004) 432-444.

[15] D. Marx, P. Blanc-Bennon, Numerical simulation of stack-heat exchangers coupling in a thermoacoustic refrigerator, AIAA Journal 42 (2004) 1338-1347.

[16] A. Piccolo, G. Pistone, Computation of the time-averaged temperature fields and energy fluxes in a thermally isolated thermoacoustic stack at low acoustic Mach numbers, International Journal of Thermal Sciences 46 (3) (2007) 235-244.

[17] L.D. Landau, E.M. Lifshitz, Fluid Mechanics, first ed., Pergamon, London, 1959.

[18] R.S. Wakeland, R.M. Keolian, Effectiveness of parallel-plate heat exchangers in thermoacoustic devices, J. Acoust. Soc. Amer. 115 (6) (2004) 2873-2886.

[19] W.H. Press, S.A. Teukolsky, W.T. Vetterling, B.P. Flannerry, Numerical recipes in Fortran, Snd. Ed., 1994, Cambridge University Press.

[20] http://www.netlib.org/lapack.

[21] P. Merkli, H. Thomann, Transition to turbulence in oscillating pipe, Journal of Fluid Mechanics 68 (1975) 567-579. 
[22] J.R. Brewster, R. Raspet, H.E. Bass, Temperature discontinuities between elements of thermoacoustic devices, Journal of the Acoustical Society of America 102 (6) (1997). [23] G.W. Swift, Analysis and performance of a large thermoacoustic engine, Journal of the Acoustical Society of America 92 (1992) 1551-1563.

[24] D. Marx, P. Blanc-Benon, Computation of the temperature distortion in the stack of a standing-wave thermoacoustic refrigerator, Journal of the Acoustical Society of America 118 (5) (2005) 2993-2999.

[25] T.J. Hofler, Effective heat transfer between a thermoacoustic heat exchanger and stack, Journal of the Acoustical Society of America 94 (1993) 1772.

[26] M.E.H. Tijani, J.C.H. Zeegers, A.T.A.M. de Waele, The optimal stack spacing for thermoacoustic refrigeration, Journal of the Acoustical Society of America 112 (1) (2002) 128-133.

[27] L. Zoontjens, C.Q. Howard, A.C. Zander, B.S. Cazzolato, Numerical study of flow and energy fields in thermoacoustic couples of non-zero thickness, International Journal of Thermal Sciences 48 (4) (2009) 733-746.

[28] E.C. Nsofor, S. Celik, X. Wang, Experimental study on the heat transfer at the heat exchanger of the thermoacoustic refrigerating system, Applied Thermal Engineering 27 (2007) 2435-2442.

[29] I. Paek, J.E. Braun, L. Mongeau, Characterizing heat transfer coefficients for heat exchangers in standing wave thermoacoustic coolers. Journal of the Acoustical Society of America, 118 (4) (2005) 2271-2280. 


\section{Figure Captions}

Figure 1. Schematic illustration of the modeled thermoacoustic refrigerator.

Figure 2. Profile of the transverse velocity amplitude $v_{y 1}$ with coordinate $y$.

Figure 3. Schematics of the HXs and stack configuration.

Figure 4. Magnified view of the computational domain (grey area).

Figure 5. Centerline plate and fins temperature as a function of coordinate $x$. In the insert the temperature discontinuities between the cold fin and the stack edge is reported as a function of drive ratio at selected $U$ values.

Figure 6. The cooling load as a function of the ratio between the temperature of the cold HX and the temperature of the hot HX at selected drive ratios. Straight lines result from a linear fit of the simulation points.

Figure 7. The cooling load as a function of the fin-to-reservoir unitary global conductance at selected drive ratios. Continuous lines are guides for the eye.

Figure 8. The mean temperature difference between the gas and cold HX fin as a function of $D R$ for two operating conditions. Dotted and continuous lines result respectively from a quadratic (low $D R$ ) and linear (high $D R$ ) fit of the simulation points. 
Figure 9. The heat loads per unit area on the cold and hot $\mathrm{HX}$ as a function of $D R$. In the insert their difference is plotted. Dotted and continuous lines result respectively from a quadratic (low $D R$ ) and linear (high $D R$ ) fit of the simulation points.

Figure 10. The cooling load as a function of the length of the cold HX at selected drive ratios. Continuous lines are guides for the eye.

Figure 11. The cooling load as a function of the plate/fin interspacing at selected $L_{C}$ values and for $D R=4.93$. Continuous lines result from the fit of the simulation points by an arbitrary peak function. The straight line passes through the locus of maxima.

Figure 12. The time-averaged transverse heat flux density at plate/fin surface $\left(y=y_{0}\right)$ as a function of the axial coordinate for two $D R$ values.

Figure 13. The mean heat flux per unit surface area exchanged by the cold fin with the gas at selected drive ratios.

Figure 14. The longitudinal profile of the time averaged transverse heat flux density at the solid surface of the cold fin at selected fin spacing.

Figure 15. The total cooling load per unit cross sectional area of the stack as a function of blockage ratio at selected lengths of the cold HX.

Figure 16. Comparison between experimental and theoretical Colburn- $j$ factors. 
Table 1. Parameters of selected simulations. In all runs test-gas $=\mathrm{He}, P_{0}=101325 \mathrm{~Pa}$, mean temperature $=300 \mathrm{~K}$, , resonance frequency $=200 \mathrm{~Hz}, U_{\text {ref }}=100 \mathrm{Wm}^{2} \mathrm{~K}^{-1}, \lambda=10.08$ $\mathrm{m}, \delta_{K}=5.352 \times 10^{-4} \mathrm{~m}, T_{H}=300 \mathrm{~K}, T_{C}=297 \mathrm{~K}, L_{S}=0.07 \mathrm{~m}$.

\begin{tabular}{|c|c|c|c|c|c|c|c|c|}
\hline Run & $y_{0} / \delta_{\kappa}$ & $l / \delta_{\kappa}$ & $x_{s} / \lambda$ & $P_{A} / P_{0}(\%)$ & $L_{C} / L_{S}$ & $L_{H} / L_{S}$ & $U_{C} / U_{r e f}$ & $U_{H} / U_{r e f}$ \\
\hline 1 & 3 & 0.93 & 0.11 & 4.93 & 0.17 & 0.18 & 1 & 1 \\
\hline 2 & 1.5 & 0.47 & 0.11 & 4.93 & 0.11 & 0.11 & 0.1 & 0.1 \\
\hline 3 & 1.5 & 0.47 & 0.11 & $0.49-5.43$ & 0.11 & 0.11 & 0.1 & 0.1 \\
\hline 4 & 1.5 & 0.47 & 0.11 & $0.49-5.43$ & 0.11 & 0.11 & 1 & 1 \\
\hline 5 & 1.5 & 0.47 & 0.11 & $0.49-5.43$ & 0.11 & 0.11 & 5 & 5 \\
\hline 6 & 1.5 & 0.47 & 0.11 & 3.95 & 0.11 & 0.11 & $0.1-20$ & $0.1-20$ \\
\hline 7 & 1.5 & 0.47 & 0.11 & 4.44 & 0.11 & 0.11 & $0.1-20$ & $0.1-20$ \\
\hline 8 & 1.5 & 0.47 & 0.11 & 4.93 & 0.11 & 0.11 & $0.1-20$ & $0.1-20$ \\
\hline 9 & 1.5 & 0.47 & 0.11 & 5.43 & 0.11 & 0.11 & $0.1-20$ & $0.1-20$ \\
\hline 10 & 1.5 & 0.47 & 0.11 & 2.47 & 0.11 & 0.11 & $0.1-20$ & $0.1-20$ \\
\hline 11 & 1.5 & 0.47 & 0.11 & 3.45 & 0.11 & 0.11 & $0.1-20$ & $0.1-20$ \\
\hline 12 & 3 & 0.93 & 0.11 & $0.49-5.92$ & 0.11 & 0.11 & 10 & 10 \\
\hline 13 & 1.5 & 0.47 & 0.11 & $0.49-5.92$ & 0.11 & 0.11 & 20 & 20 \\
\hline 14 & 1.5 & 0.47 & 0.11 & $0.49-7.90$ & 0.11 & 0.11 & 20 & 20 \\
\hline 15 & 1.5 & 0.47 & 0.11 & 2.96 & $0.0143-0.343$ & 0.18 & 30 & 30 \\
\hline 16 & 1.5 & 0.47 & 0.11 & 4.93 & $0.0143-0.343$ & 0.18 & 30 & 30 \\
\hline 17 & 1.5 & 0.47 & 0.11 & 6.91 & $0.0143-0.343$ & 0.18 & 30 & 30 \\
\hline 18 & $0.5-3$ & $0.16-0.93$ & 0.11 & 4.93 & 0.0143 & 0.18 & 30 & 30 \\
\hline 19 & $0.5-3$ & $0.16-0.93$ & 0.11 & 4.93 & 0.036 & 0.18 & 30 & 30 \\
\hline 20 & $0.5-3$ & $0.16-0.93$ & 0.11 & 4.93 & 0.071 & 0.18 & 30 & 30 \\
\hline 21 & $0.5-3$ & $0.16-0.93$ & 0.11 & 4.93 & 0.126 & 0.18 & 30 & 30 \\
\hline 22 & $0.5-3$ & $0.16-0.93$ & 0.11 & 4.93 & 0.180 & 0.18 & 30 & 30 \\
\hline 23 & $0.5-3$ & $0.16-0.93$ & 0.11 & 4.93 & 0.343 & 0.18 & 30 & 30 \\
\hline 24 & 2 & 0.8 & 0.11 & 1.63 & 0.14 & 0.14 & 20 & 20 \\
\hline 25 & 2 & 0.8 & 0.11 & 6.91 & 0.14 & 0.14 & 20 & 20 \\
\hline 26 & $0.5-3$ & 0.75 & 0.11 & 4.93 & 0.036 & 0.18 & 30 & 30 \\
\hline 27 & $0.5-3$ & 0.75 & 0.11 & 4.93 & 0.018 & 0.18 & 30 & 30 \\
\hline 28 & $0.5-3$ & 0.75 & 0.11 & 4.93 & 0.343 & 0.18 & 30 & 30 \\
\hline 29 & 1.5 & 0.47 & 0.09 & 7.9-8.9 & 0.29 & 0.29 & 20 & 20 \\
\hline 30 & 1.5 & 0.47 & $0.09-0.08$ & 8.9 & 0.29 & 0.29 & 20 & 20 \\
\hline 31 & 1.5 & 0.47 & 0.07 & 9.9 & 0.29 & 0.29 & 20 & 20 \\
\hline 32 & 1.5 & 0.47 & 0.11 & $0.99-7.89$ & 0.11 & 0.11 & 20 & 20 \\
\hline 33 & 1.5 & 0.47 & $0.1-0.09$ & 7.89 & 0.11 & 0.11 & 20 & 20 \\
\hline 34 & 2.0 & 0.8 & 0.11 & $0.69-8.0$ & 0.14 & 0.14 & 20 & 20 \\
\hline 35 & 2.0 & 0.8 & $0.11-0.1$ & 8.0 & 0.14 & 0.14 & 20 & 20 \\
\hline 36 & 2.0 & 0.8 & $0.09-0.07$ & 8.0 & 0.43 & 0.43 & 20 & 20 \\
\hline 37 & 2.0 & 0.8 & 0.07 & 8.4-10.8 & 0.43 & 0.43 & 20 & 20 \\
\hline
\end{tabular}




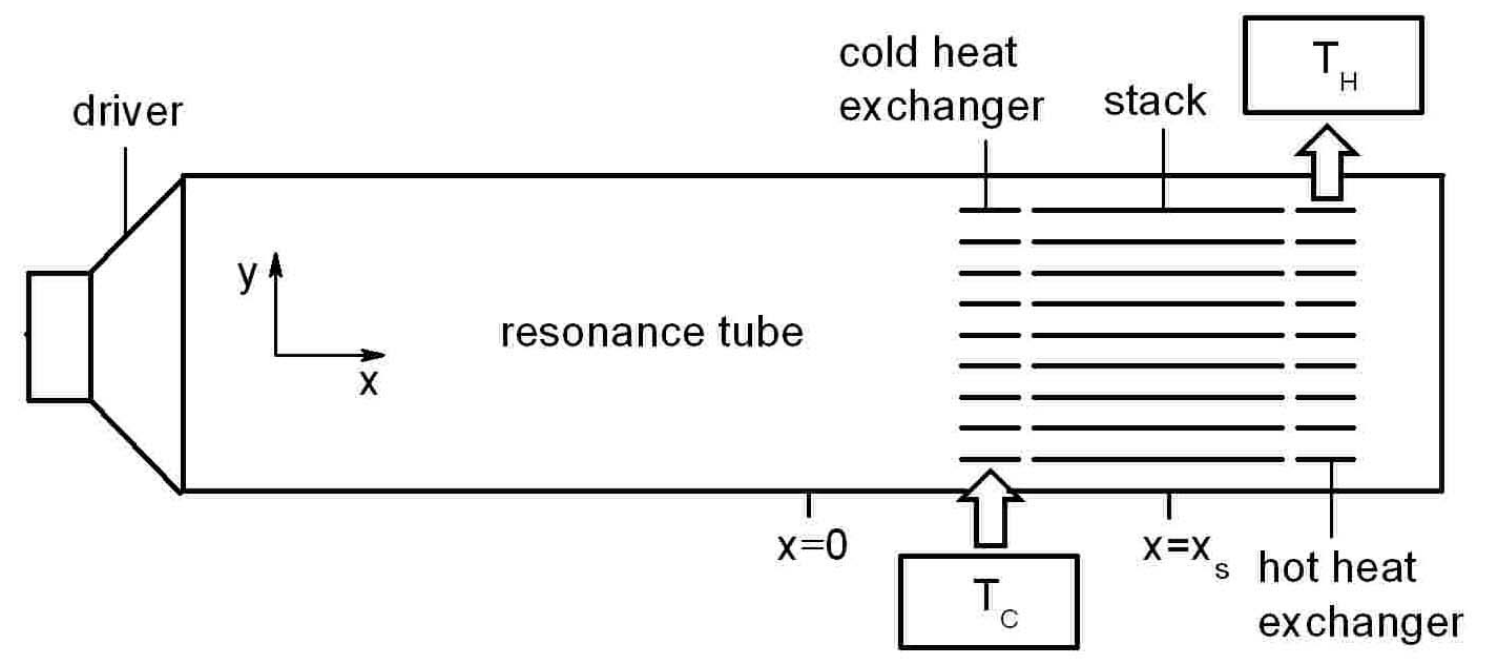

Figure 1

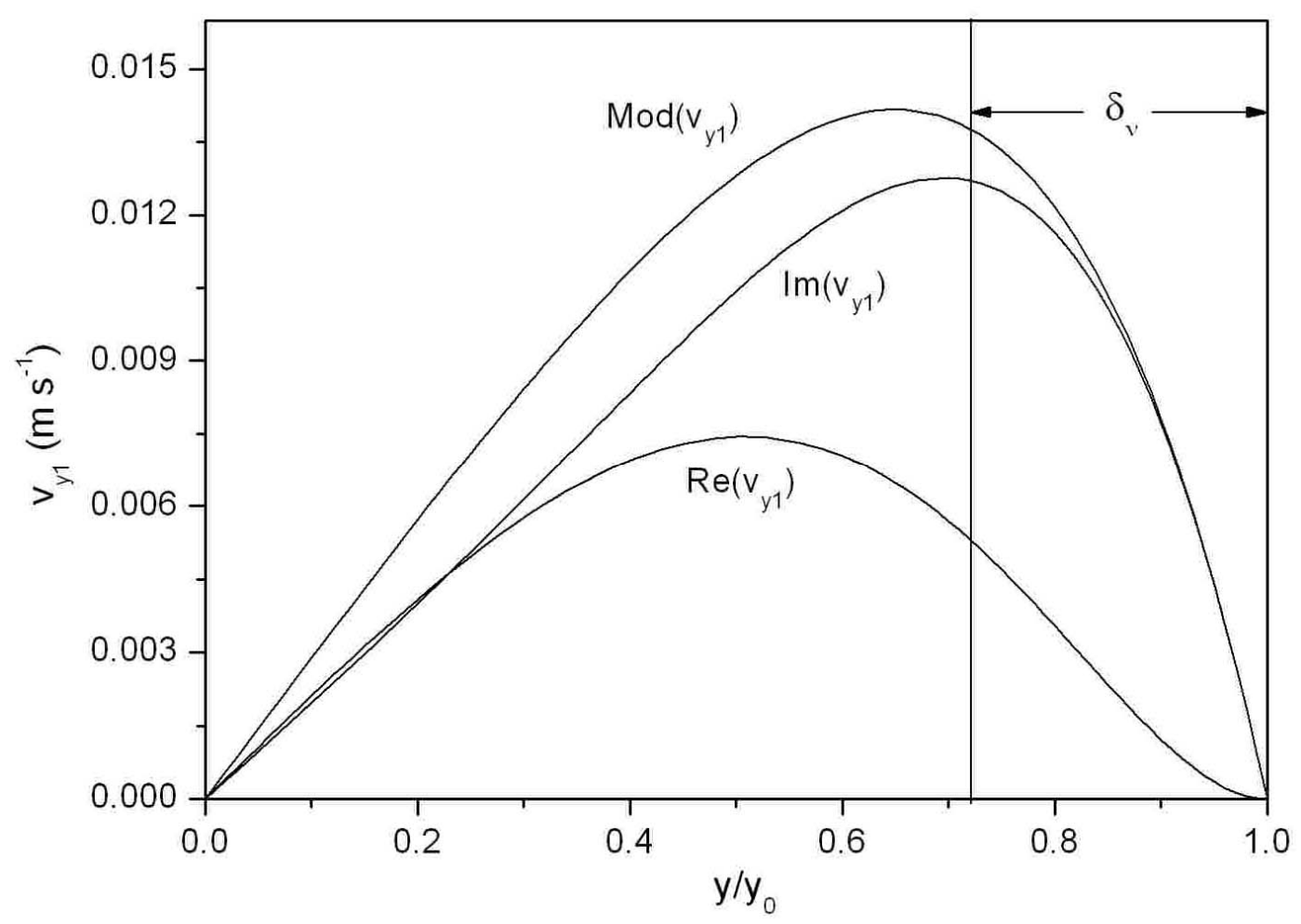

Figure 2 


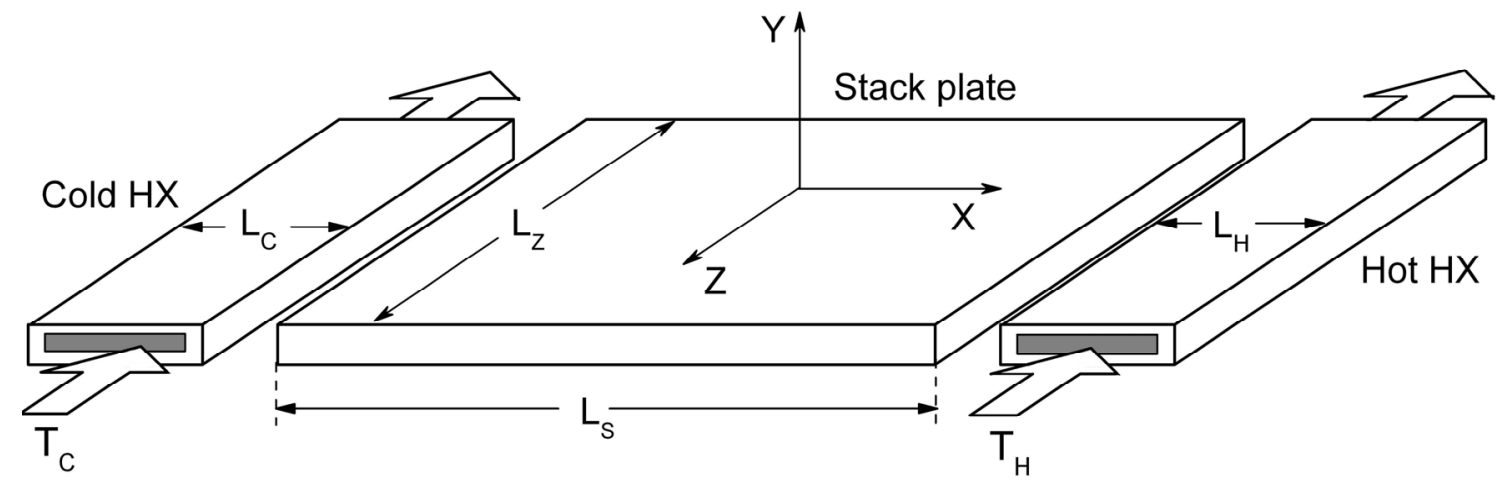

Figure 3

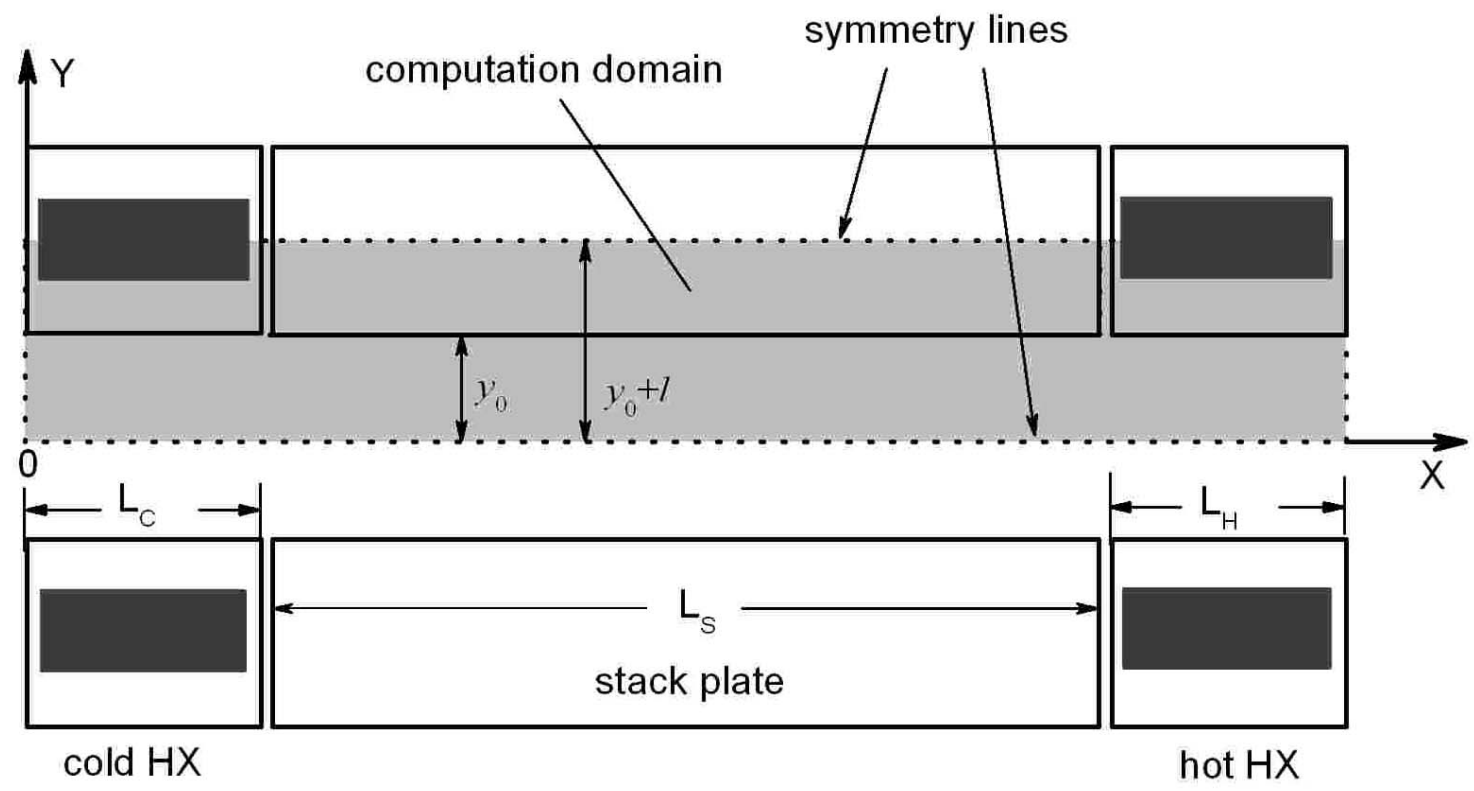

Figure 4 


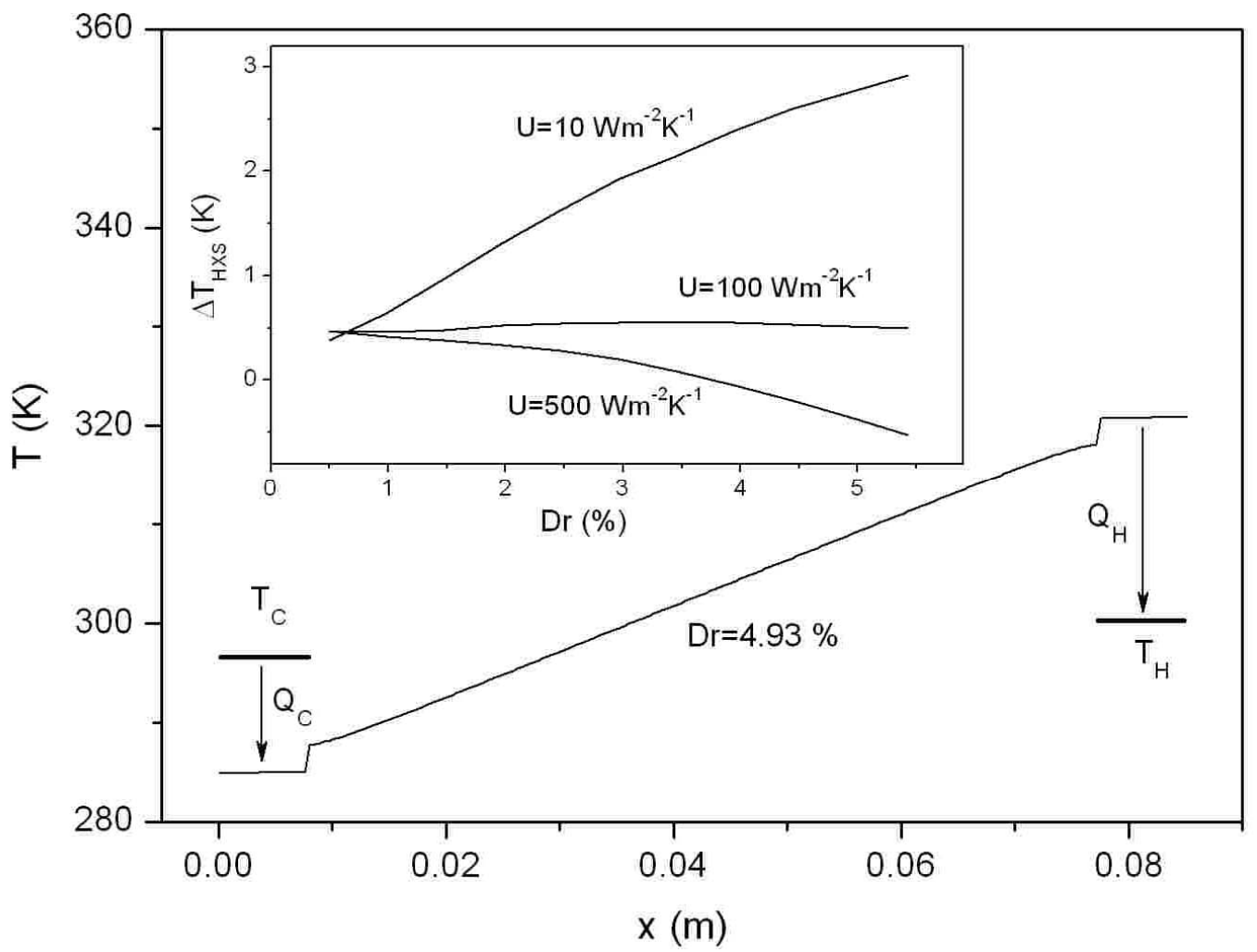

Figure 5

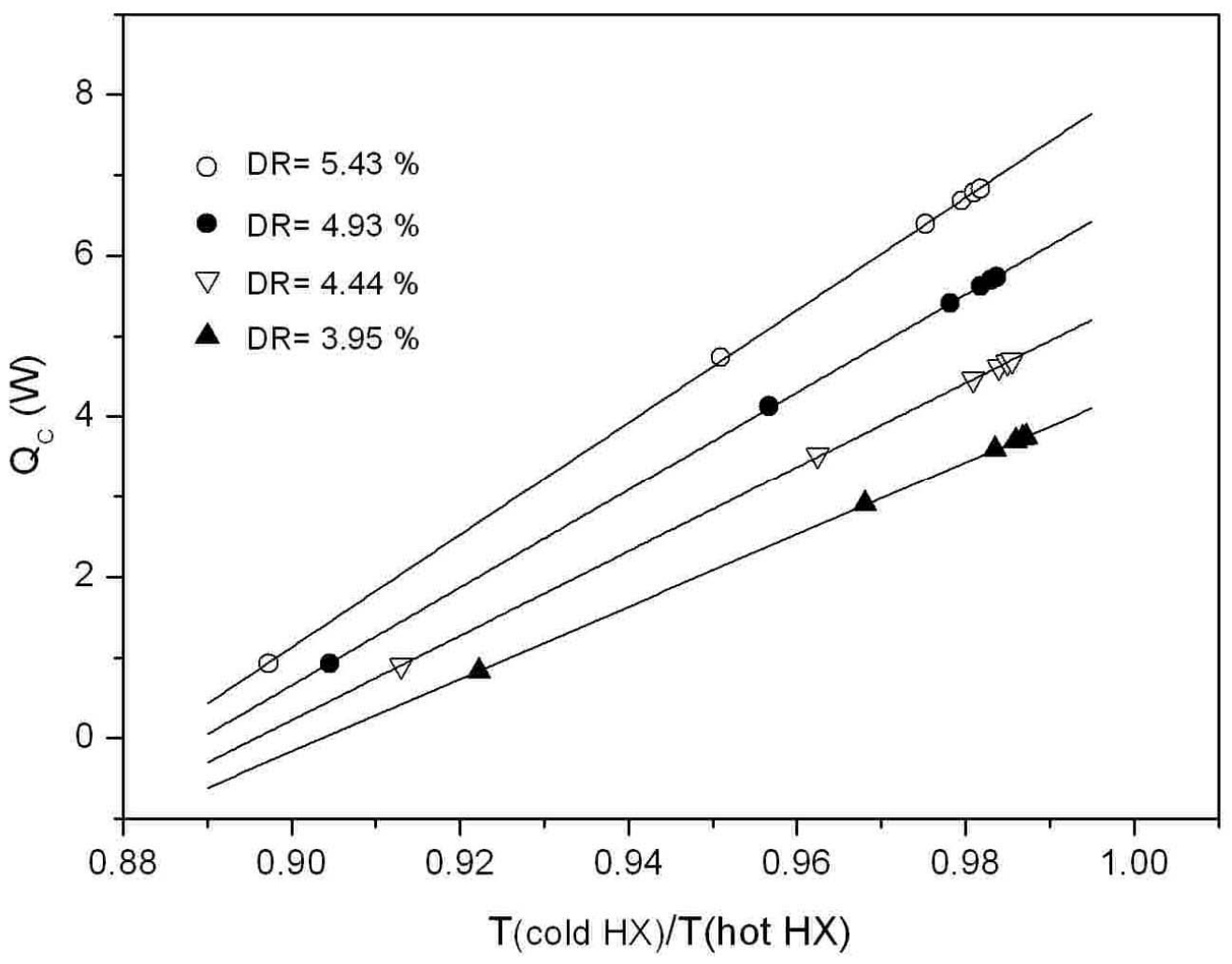

Figure 6 


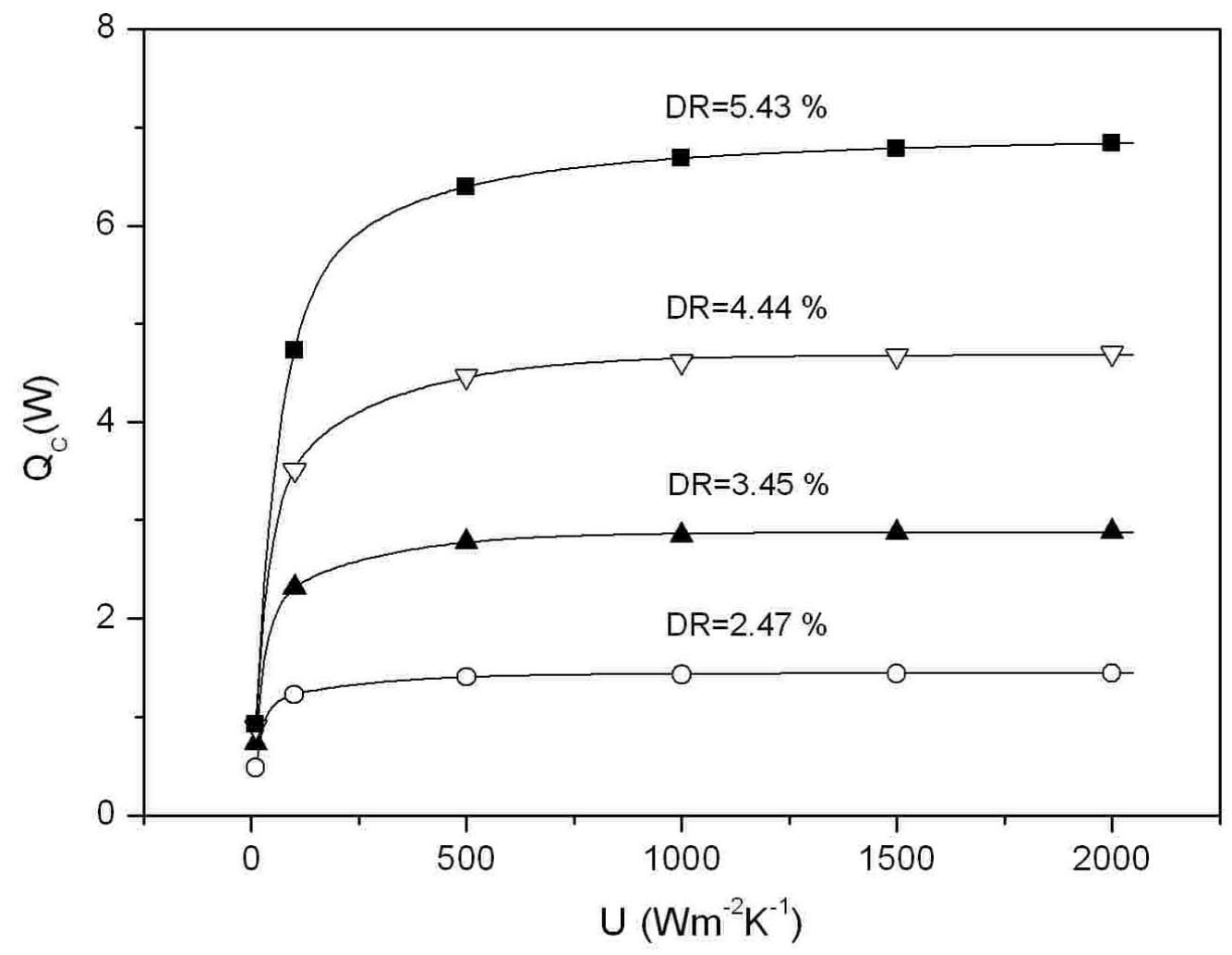

Figure 7

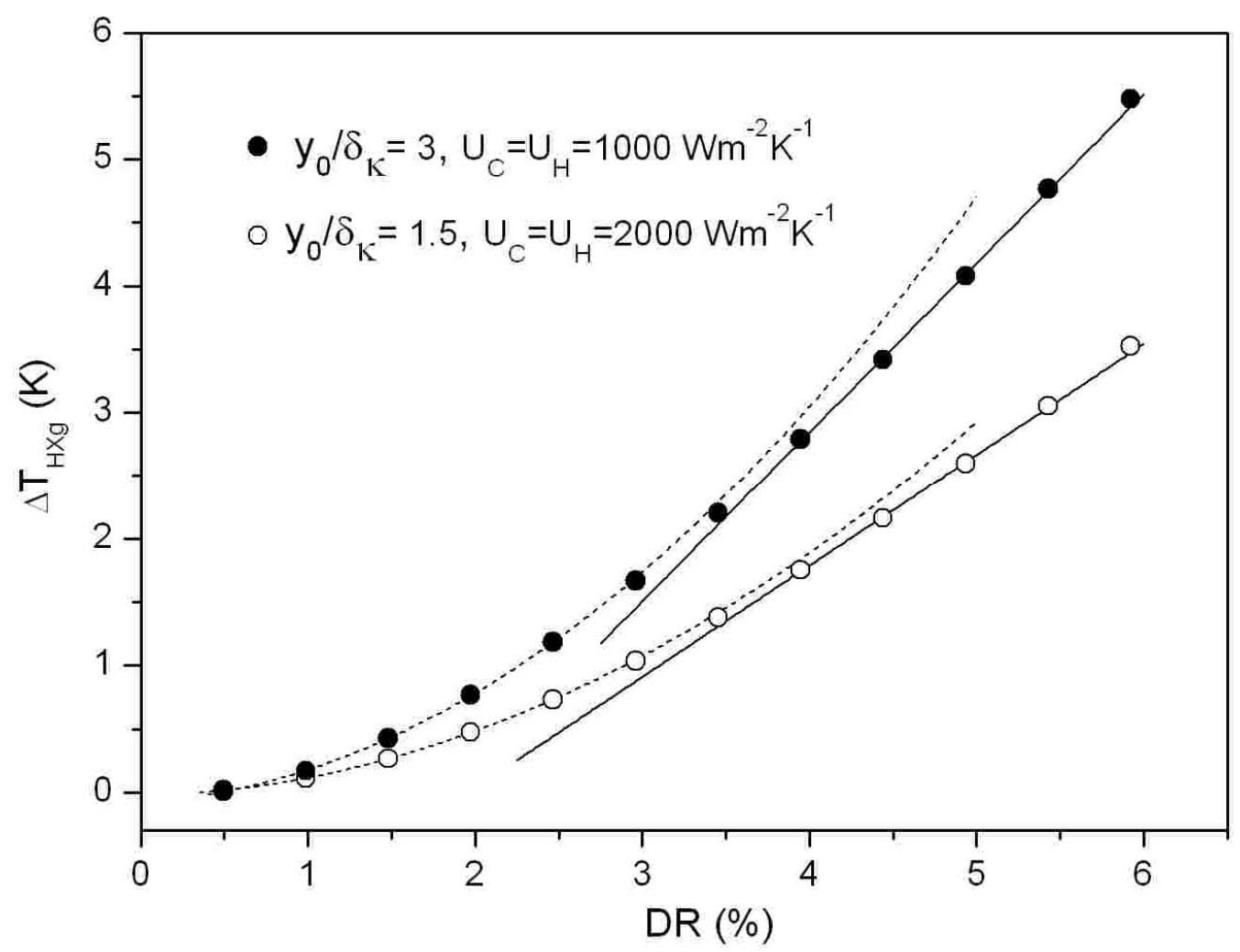

Figure 8 


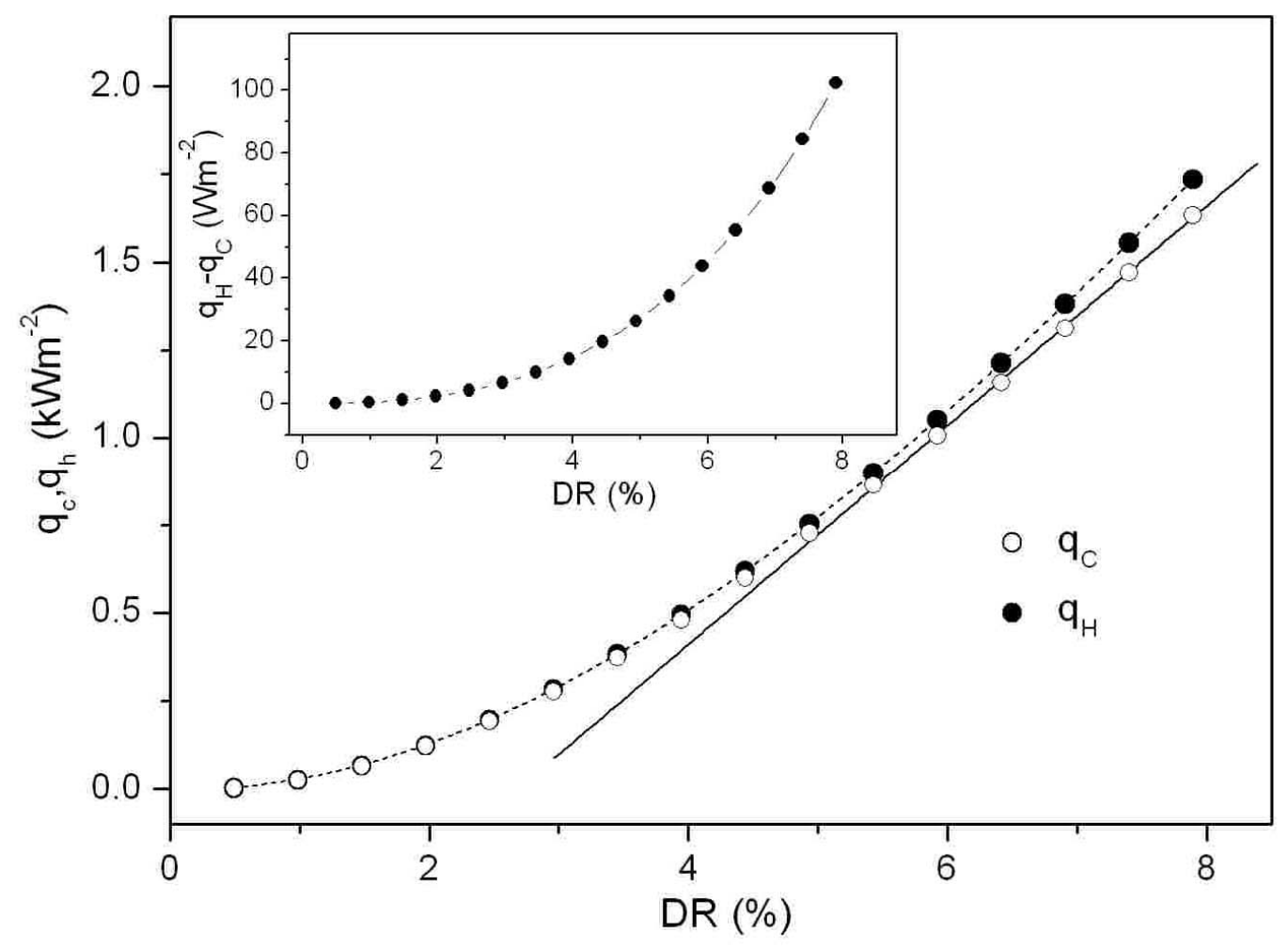

Figure 9

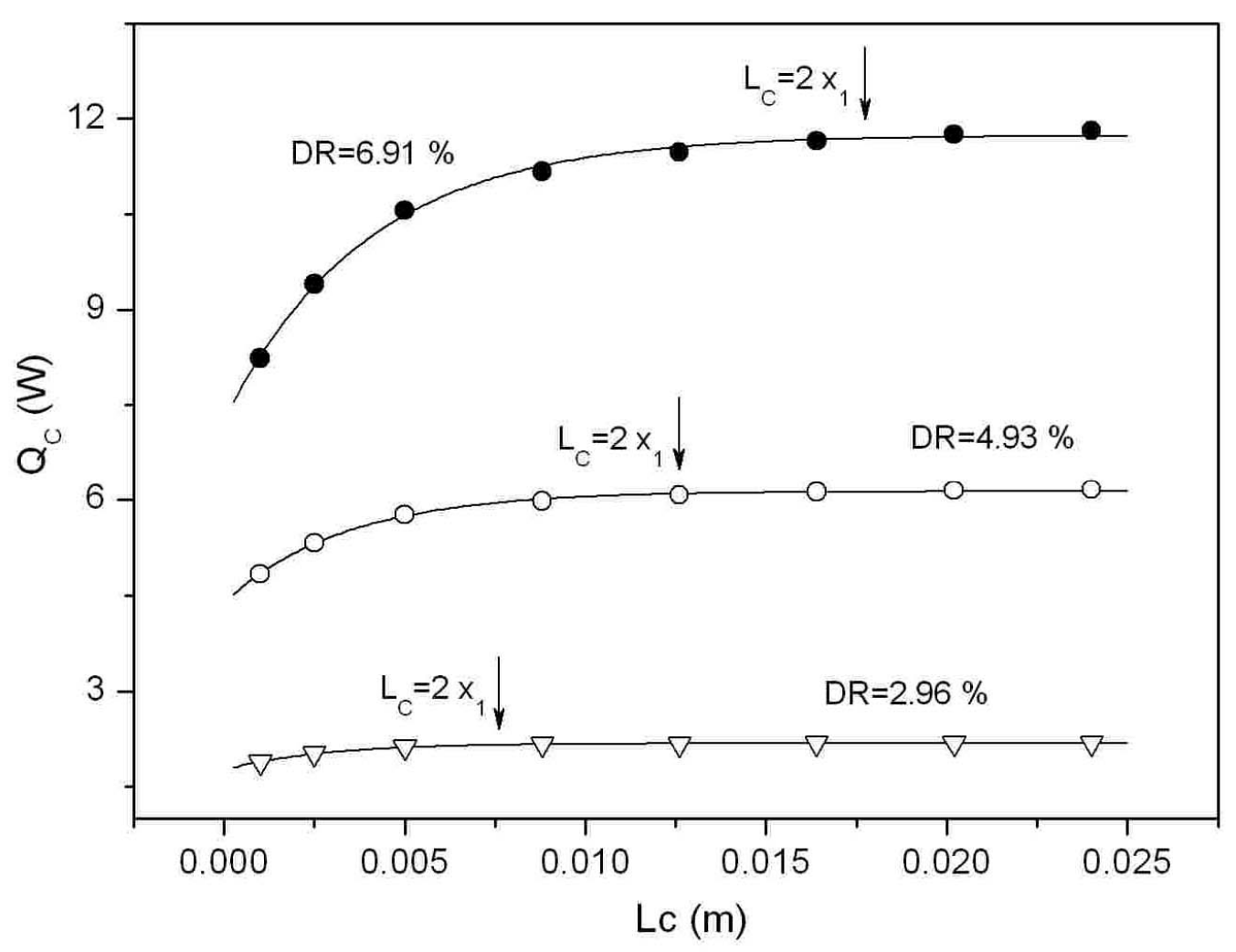

Figure 10 


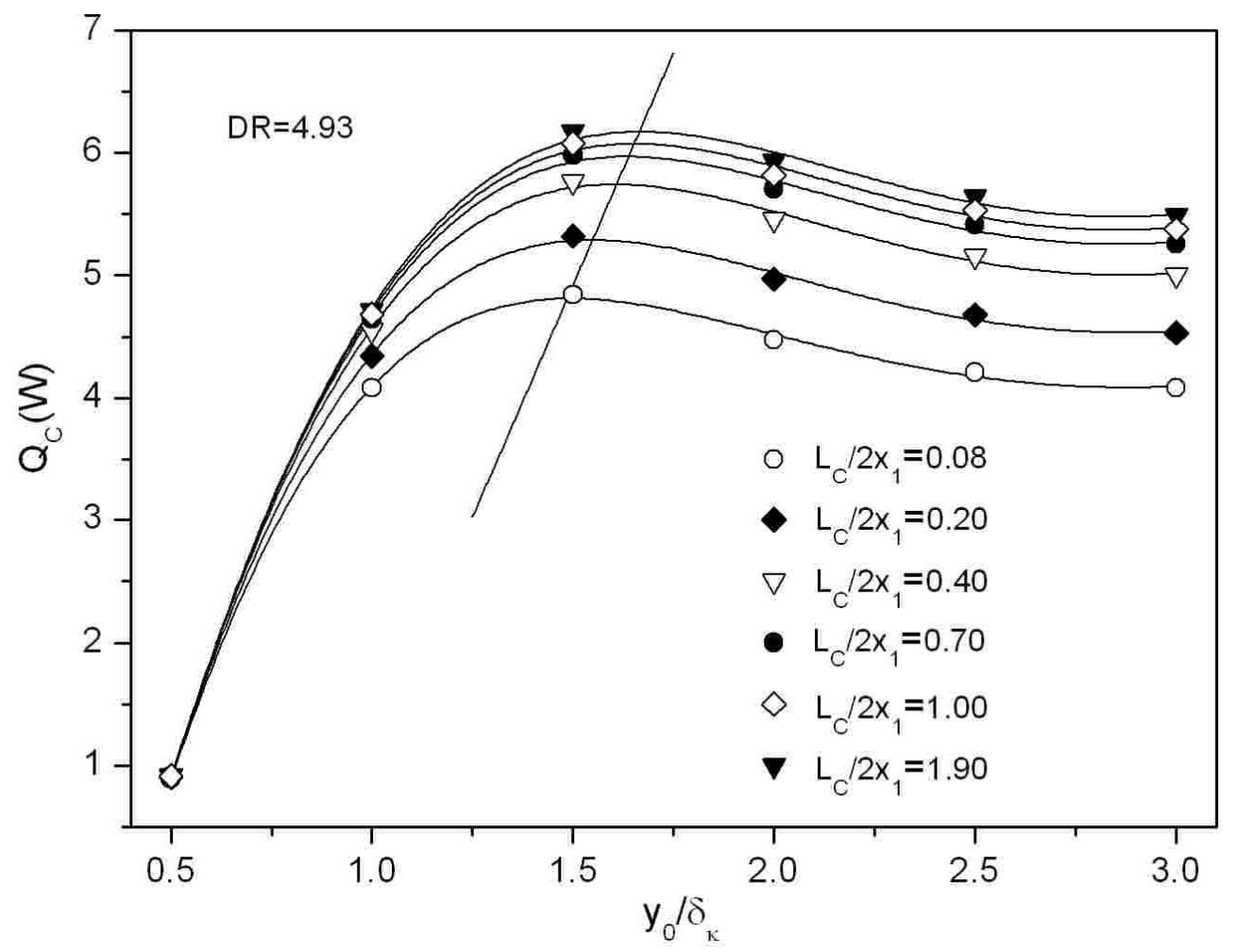

Figure 11

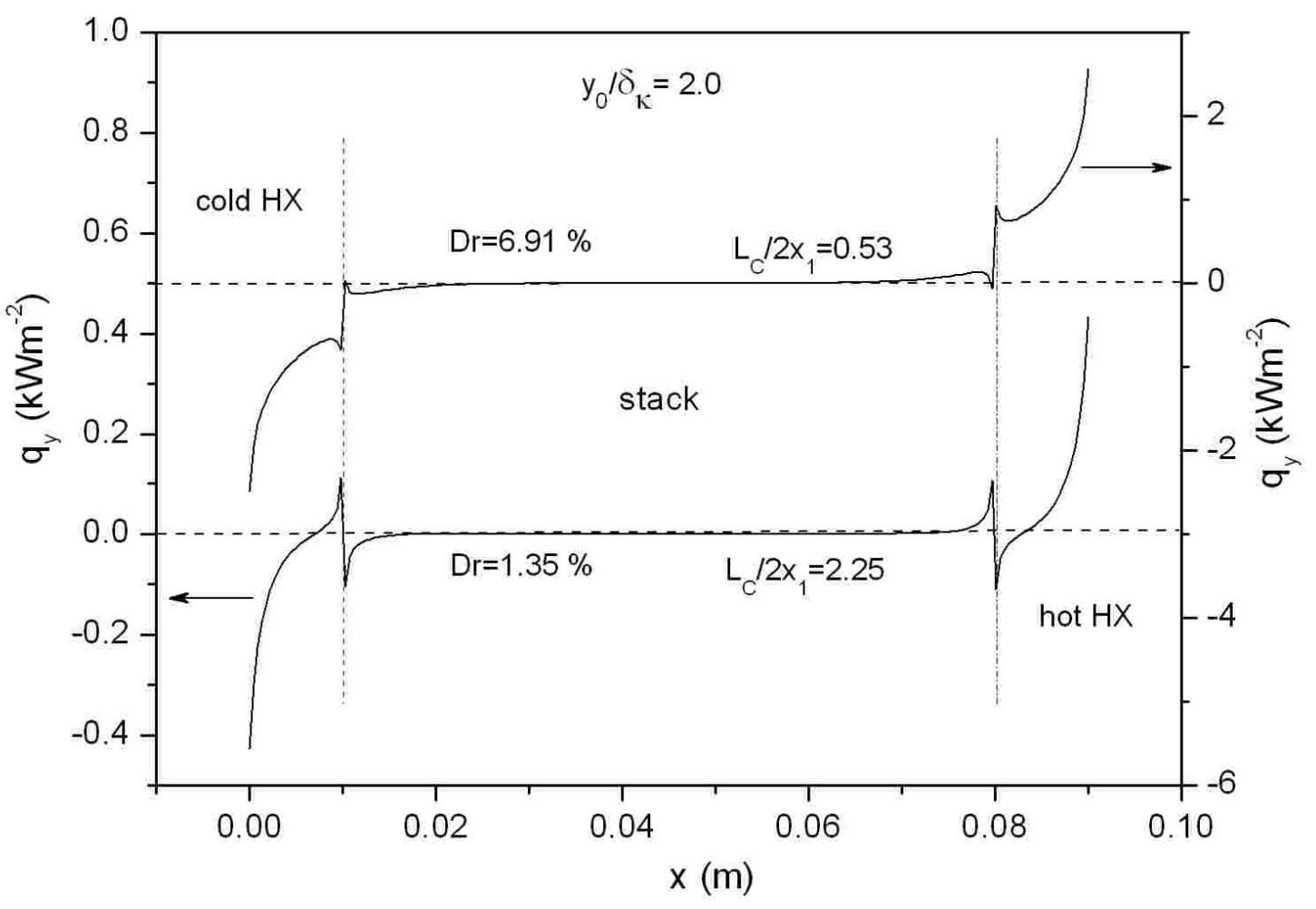

Figure 12 


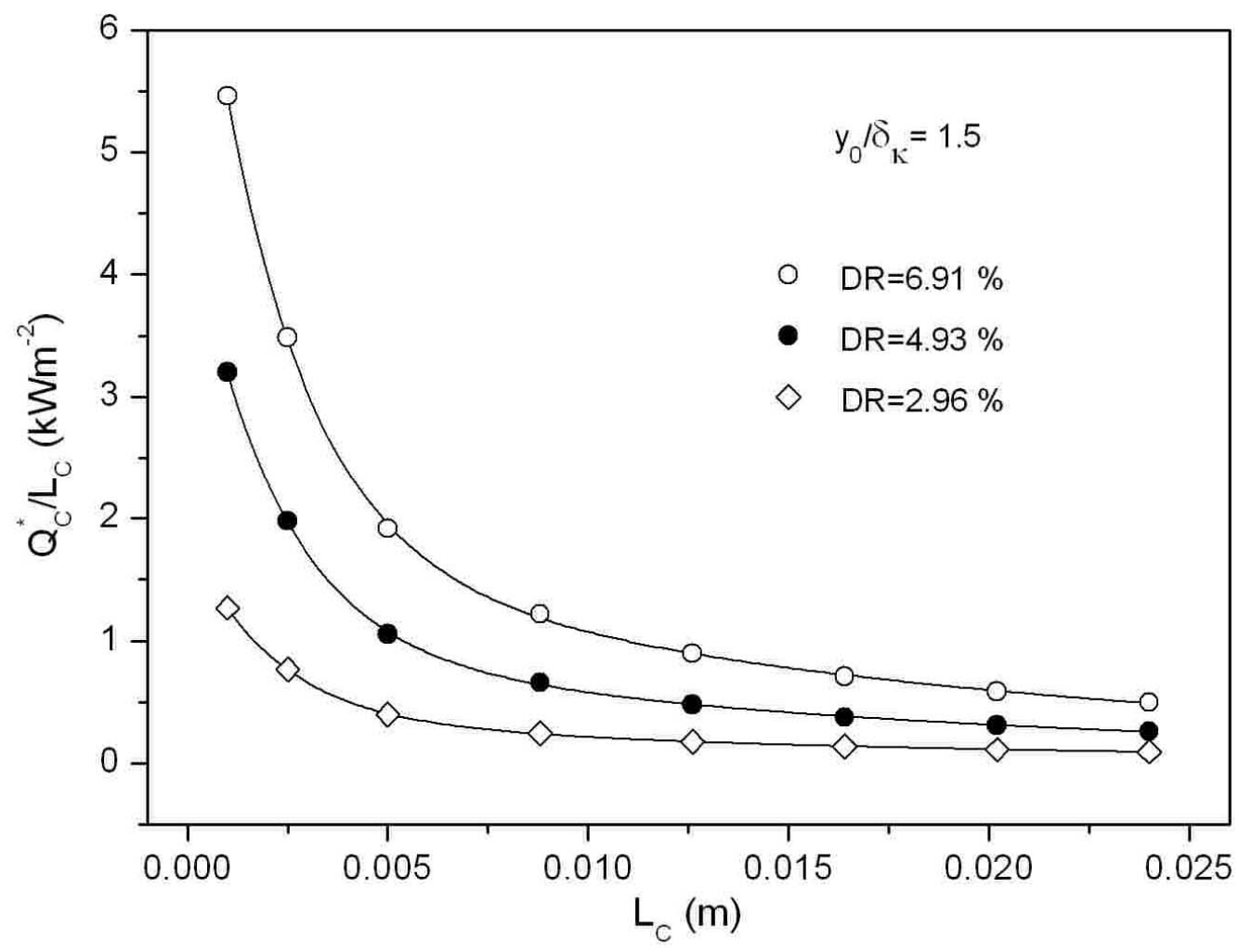

Figure 13

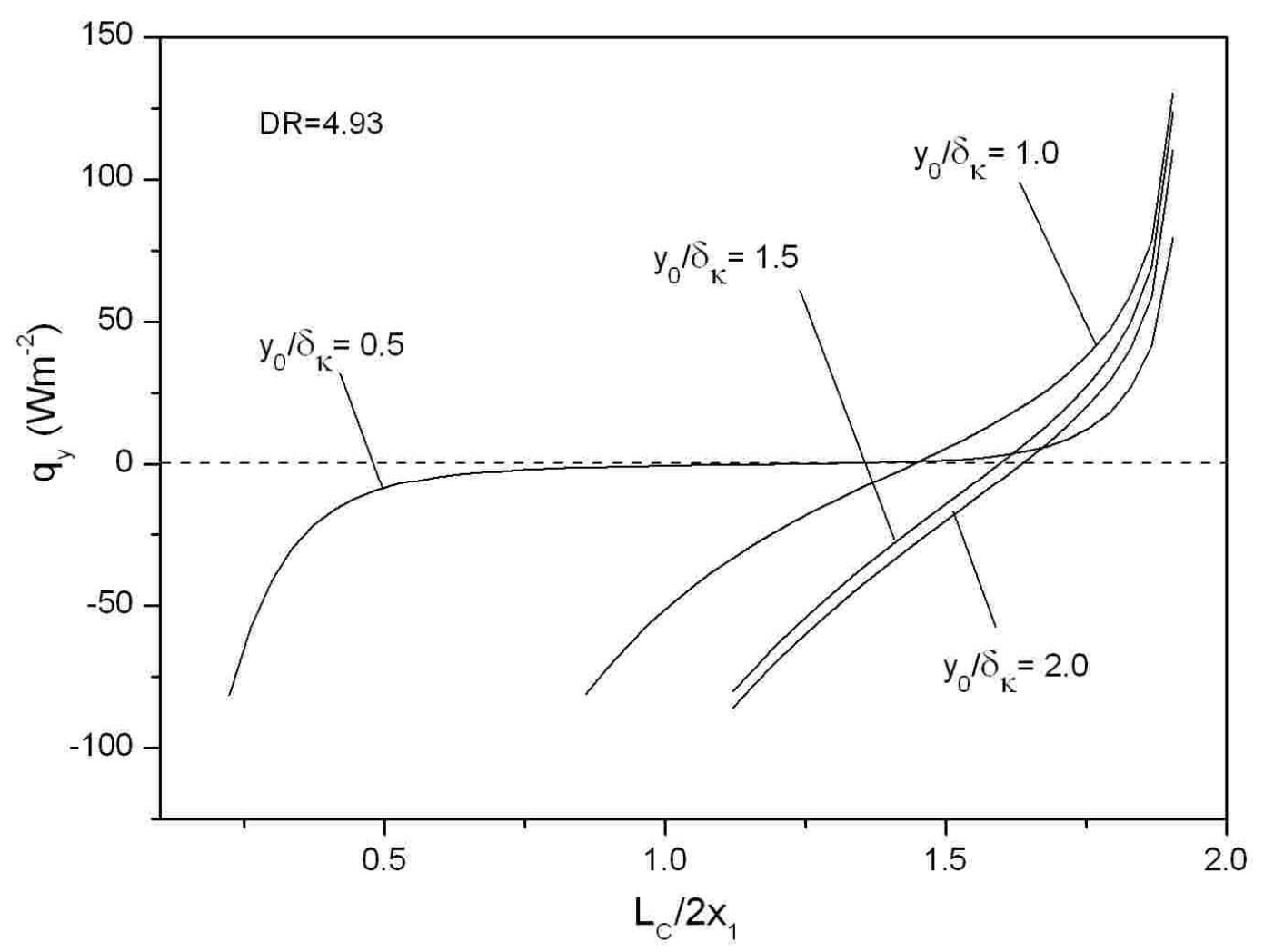

Figure 14 


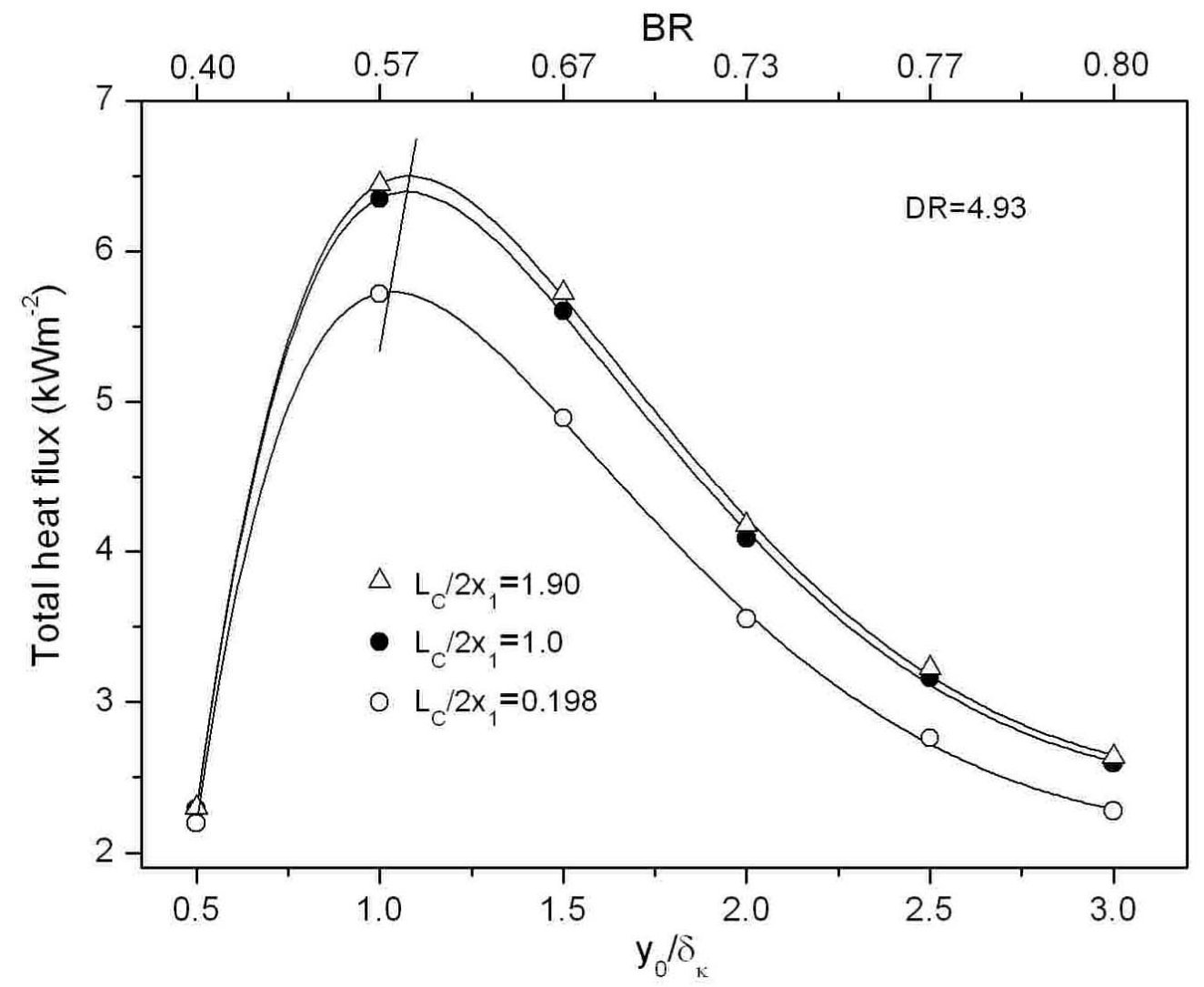

Figure 15

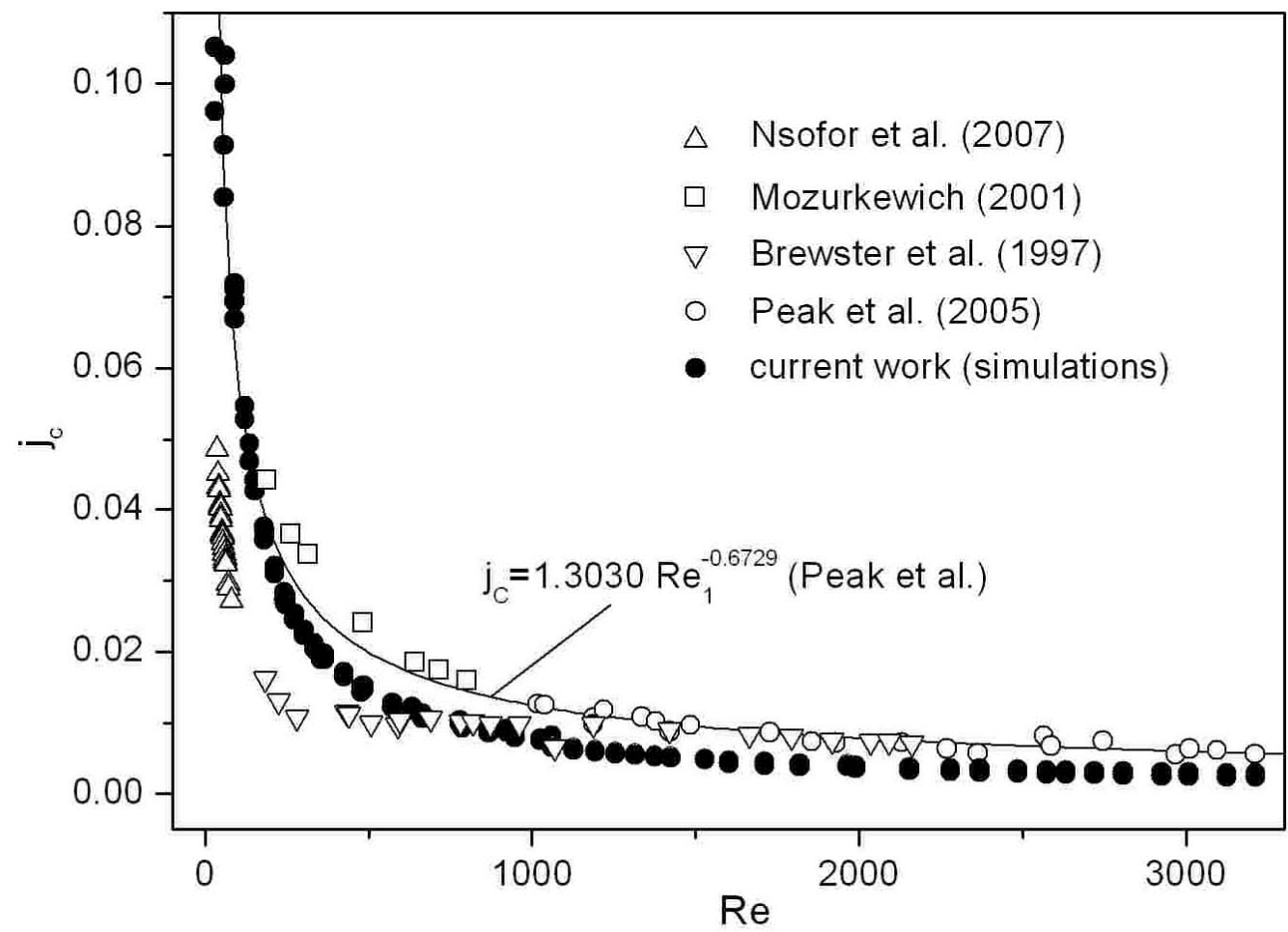

Figure 16 